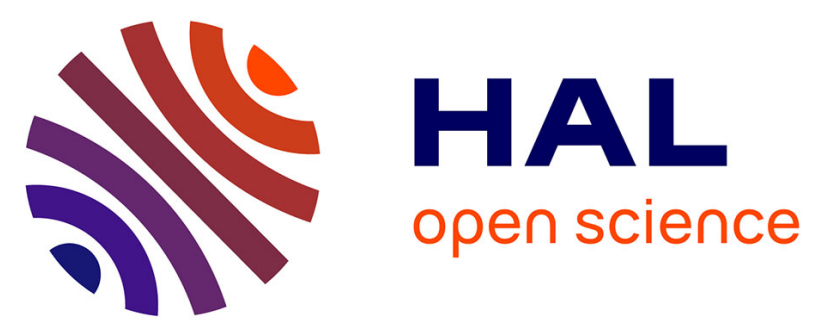

\title{
Biochemical and Structural Characterization of TesA, a Major Thioesterase Required for Outer-Envelope Lipid Biosynthesis in Mycobacterium tuberculosis
}

Phuong Chi Nguyen, van Son Nguyen, Benjamin Martin, Patrick Fourquet, Luc Camoin, Chistopher Spilling, Jean-François Cavalier, Christian Cambillau, Stéphane Canaan

\section{To cite this version:}

Phuong Chi Nguyen, van Son Nguyen, Benjamin Martin, Patrick Fourquet, Luc Camoin, et al.. Biochemical and Structural Characterization of TesA, a Major Thioesterase Required for Outer-Envelope Lipid Biosynthesis in Mycobacterium tuberculosis. Journal of Molecular Biology, 2018, 430 (24), pp.5129-5136. 10.1016/j.jmb.2018.09.017 . hal-01894053

\section{HAL Id: hal-01894053 \\ https://hal-amu.archives-ouvertes.fr/hal-01894053}

Submitted on 29 Jan 2020

HAL is a multi-disciplinary open access archive for the deposit and dissemination of scientific research documents, whether they are published or not. The documents may come from teaching and research institutions in France or abroad, or from public or private research centers.
L'archive ouverte pluridisciplinaire HAL, est destinée au dépôt et à la diffusion de documents scientifiques de niveau recherche, publiés ou non, émanant des établissements d'enseignement et de recherche français ou étrangers, des laboratoires publics ou privés. 


\section{Biochemical and structural characterization of TesA, a major thioesterase required for phthiocerol dimycocerosates and phenolic glycolipids biosynthesis in M. tuberculosis}

Phuong Chi Nguyen", Van Son Nguyen ${ }^{\mathbb{I}}$, Benjamin P. Martin ${ }^{ \pm}$, Patrick Fourquet ${ }^{\S}$, Luc Camoin $^{\S}$, Chistopher D. Spilling ${ }^{ \pm}$, Jean-François Cavalier ${ }^{\#}$, Christian Cambillau ${ }^{\mathbb{I}}$ and Stéphane Canaan $^{\#, *}$

\#Aix Marseille Univ, CNRS, LISM, IMM FR3479, Marseille, France

"IAix Marseille Univ, CNRS, AFMB, Marseille, France

${ }^{\S}$ Aix Marseille Univ, CNRS, INSERM, Institut Paoli-Calmettes, CRCM, Marseille Protéomique, Marseille, France

${ }^{ \pm}$Department of Chemistry and Biochemistry, University of Missouri, One University Boulevard, St. Louis, MO 63121, USA

*Corresponding author: stephane.canaan@imm.cnrs.fr, phone +33 491164093

Short title: $M$. tuberculosis TesA 3D structure

\begin{abstract}
Due to the high number of patients infected by tuberculosis (TB) and the sharp increase of drug resistant TB cases, developing new drugs to fight this disease has become increasingly urgent. In this context, a new class of compound, analogs of the naturally occurring enolphosphates Cyclipostins and Cyclophostin (CyC analogs), offer new therapeutic opportunities. The $\mathrm{CyC}$ analogs display potent activity both in vitro and in infected macrophages against several pathogenic mycobacteria including Mycobacterium tuberculosis and Mycobacterium abscessus. Interestingly, these $\mathrm{CyC}$ inhibitors target several enzymes with active site serine or cysteine residues that play key roles in mycobacterial lipid and cell wall metabolism. Among them, TesA, a putative thioesterase involved in the synthesis of phthiocerol dimycocerosates (PDIMs) and phenolic glycolipids (PGLs), has been identified. These two lipids (PDIMS and PPGLs) are non-covalently bound to the outer cell wall in several human pathogenic mycobacteria and are important virulence factors. Herein, we used biochemical and
\end{abstract}

structural approaches to validate TesA as an effective pharmacological target of the CyC analogs. We have confirmed both thioesterase and esterase activities of TesA, and have shown that the most active inhibitor $\mathrm{CyC}_{17}$ binds covalently to the catalytic Ser104 residue leading to a total loss of enzyme activity. These data were supported by the X-ray structure, obtained at a $2.6 \AA$ resolution, of a complex in which $\mathrm{CyC}_{17}$ is bound to TesA . Our study provides evidence that $\mathrm{CyC}_{17}$ inhibits the activity of TesA, thus paving the way to a new strategy for impairing the PDIM and PGL biosynthesis, potentially decreasing the virulence of associated mycobacterial species.

Keywords: lipids, virulence, Cyclipostins, Cyclophostin, PDIM, PGL, thioesterase inhibitor

\section{Introduction}

Mycobacterium tuberculosis (M. tuberculosis) the causative agent of tuberculosis (TB) is responsible for an estimated 10.4 million new cases and 1.7 million deaths across the globe, as reported by WHO in 2017 [1]. With the emergence 
of multi and extensive drug resistance strains, TB remains the leading cause of death from an infectious disease. Due to the very complex composition of $M$. tuberculosis cell wall and the capacity of the bacilli to hide from the immune system inside granulomas, it is challenging to efficiently treat the disease. Indeed, the $M$. tuberculosis cell wall is composed of a large number of hydrophobic molecules having up to 90 carbon atoms chain length. This thick lipid rich structure is responsible for the high impermeability of the cell wall and thus to the inherent resistance of $M$. tuberculosis to numerous macromolecules including many antibiotics [2]. Among these outer membrane/surface-exposed hydrophobic lipids, are the phthiocerol dimycocerosate (PDIM), and a second group of glycosylated compounds known as phenolic glycolipids (PGLs) and named phenolphthiocerol dimycocerosates. These two complex lipids, esterified with multimethyl-branched long-chain fatty acids, are non-covalently bound to other cell wall components and have been shown to be important virulence factors [3]. Consistent with their main role in the permeability of the cell envelope and pathogenicity [4], PDIMs are apparently produced by all virulent clinical isolates of M. tuberculosis. In contrast, the ability to synthesize PGL has only been retained by $M$. canettii and some $M$. tuberculosis isolates of the East Asian/Beijing lineage [5-8]. As an example, it has been shown that PGLs could be in part responsible for the resistance to antibiotics of $M$. tuberculosis strains of the W-Beijing family [8, 9]. Moreover, the virulence of PGL/PDIM deficient-bacteria was strongly attenuated in a mouse model suggesting a major impact of these lipids on the viability of the mycobacteria in in vivo conditions [8-12]. Hence, deciphering the PDIM and the PGL biosynthesis pathway could be helpful to learn more about the cell wall assembly of clinical pathogenic bacteria, and to find new specific therapeutic targets.
New antibiotics targeting one or more proteins involved in the synthesis of mycobacterial lipids would thus represent a promising way to fight and control TB [9, 13]. In this context, monocyclic enolphosphate and phosphonate analogs (CyC) of natural Cyclipostins and Cyclophostin [14-17] represent a new family of potent and selective inhibitors against mycobacteria only [18]. These $\mathbf{C y C}$ analogs act as powerful antitubercular agents affecting growth of $M$. tuberculosis both in vitro and in infected macrophages with very low toxicity towards mammalian host cells [19]. To identify the putative target(s) of the best inhibitor $\mathbf{C y C}_{\mathbf{1 7}}$, an activity-based protein profiling (ABPP) approach was used, leading to the capture of several active Ser- and Cys-containing enzymes in a complex proteome [19]. Among the 23 proteins identified via mass spectrometry experiments [19], TesA a putative thioesterase (annotated Rv2928) proposed to be involved in the biosynthesis pathway of both PDIM and PGL [10, 20] has been identified.

Tes A, which is localized in the membrane fraction of the bacteria [21, 22], is non-essential for the in vitro growth of $M$. tuberculosis. However, the virulence of a $\triangle t e s A$ deletion mutant strain of $M$. tuberculosis was strongly attenuated in infected cells, becoming more susceptible to antibiotics $[9,10,20,23]$. This result has been attributed to the fact that the $\Delta t e s A$ mutant in $M$. tuberculosis $\mathrm{H} 37 \mathrm{Rv}$ was defective in PDIMs [24]. More recently, TesA has been proposed to release phtiocerol and phenolpthiocerol molecules that further condense with mycoserosic acid by the action of the PapA5 enzyme to form PDIM as well as PGL skeletons [9, 10]. Thus M. tuberculosis TesA represents a major contributing partner in the synthesis of both PDIM and PGL by interacting with PpsE inside the polyketide synthase cluster $[10,25]$ (Figure 1). Therefore, this pathway may represent an interesting source of new targets for the development of novel TB 
drugs, with particular relevance to the emergence of drug resistant strains.

Consequently, M. tuberculosis TesA is believed to play an important role in vivo by increasing the virulence of pathogenic mycobacteria. However, although many $e x$ vivo experiments have been reported to decipher the physiological role of TesA, little or no information is available regarding its biochemical and structural characterization.

The present study was undertaken to decipher, the selective inhibition of TesA activity by the $\mathbf{C y C}_{\mathbf{1 7}}$ inhibitor using a combination of biochemical and structural approaches. Remarkably, we report here the first crystal structure of this M. tuberculosis enzyme in complex with the $\mathbf{C y C}_{\mathbf{1 7}}$ covalently bound to the active site Ser104 residue. This crystal structure may help optimizing new $\mathbf{C y C}$ scaffolds with higher specificity and potency against $M$. tuberculosis.

\section{Results}

\section{Expression and purification of recombinant TesA and mutant}

Both TesA and TesA ${ }^{\text {S104A }}$ were produced in recombinant forms and purified to affinity, leading to 5-10 $\mathrm{mg}$ of pure recombinant proteins per liter of culture. Their purity as well as the expected molecular weight ( $29 \mathrm{kDa})$ was confirmed by $12 \%$ SDS-PAGE (Figure 2A). TesA as well as TesA ${ }^{\text {S104A }}$ were concentrated up to $15 \mathrm{mg} / \mathrm{mL}$, the highest concentration used for crystallographic experiments (Figure 2A).

TesA has been previously predicted to be a thioesterase [20]. However, it has been reportedthat this family of enzymes can also act as esterases, phospholipases, lysophospholipases as well as lipases [26]. To investigate whether TesA exhibits such a diverse activity, a broad range of substrates have been tested including Palmitoyl-coenzyme A, para-nitrophenyl $(p \mathrm{NP})$ esters carrying various carbon chain lengths, phospholipids and triacylglycerols.
As a control for phospholipase activity we used the Cutinase-like protein Cut6 (Rv3802c), a Pks13-associated thioesterase proposed to be involved in mycolic acid biosynthesis $[27,28]$. TesA did not express any phospholipase, lysophospholipase, nor triacylglycerol lipase activities. However, TesA displayed hydrolytic activity on ester substrates (Figure 2B) being more active on $p \mathrm{NP}$ esters with short carbon chain lengths $(\mathrm{C} 2-\mathrm{C} 8)$ than with the those bearing medium and long carbon chain lengths (i.e., C12-C18). Indeed, the hydrolysis capacity of TesA decreased with increasing alkyl chain lengths. Moreover, in all cases, the presence of Triton X-100 led to a significant increase in the lipolytic activity of TesA, from 2-fold with $p$ NP-C2 and up to 127fold when using $p$ NP-C16. Under these conditions, $p$ NP-C2 having the shortest carbon chain length showed a maximum specific activity of $257 \pm 3.3 \mathrm{mU} / \mathrm{mg}$. However, with $p \mathrm{NP}$ esters carrying more than 8 carbon atoms, this specific activity dropped sharply to $43 \pm 1.9,23 \pm 0.3$ and 15 $\pm 0.8 \mathrm{mU} / \mathrm{mg}$ with $p \mathrm{NP}-\mathrm{C} 12, p \mathrm{NP}-\mathrm{C} 14$ and $p \mathrm{NP}-\mathrm{C} 16$, respectively. High variations in the enzyme activity induced by the presence of surfactant (i.e., Triton X-100) have been associated with a conformational change of the enzyme in response to the new environment but without losing its catalytic properties. More precisely, such modifications often occur when a lid covering the active site is present. It is now well established in the case of lipolytic enzymes, that surfactants can both help in solubilizing long chains substrates by forming mixed micelles, but can also promote and stabilize the lid opening process making the active site accessible to solvent [29]. As a consequence of such lid opening, an enhanced enzyme activity will be obtained, as observed with TesA.

Notably, this latter $p \mathrm{NP}$ assay also allows the discrimination of esterases from true lipases, which hydrolyze short-chains esters, and medium- to long-chain esters, respectively [30]. Here, the absence of lipase activity indicates that TesA behaves 
as an esterase, in contrast to LipY or cfp21, the only $M$. tuberculosis true triacylglycerol lipases characterized so far $[31,32]$.

As expected from the genome annotation and previous in vivo experiment $[10,20]$, TesA also exhibited thioesterase activity with a specific activity of 6.82 $\mathrm{mU} / \mathrm{mg}$ on Palmitoyl-CoA. It is noteworthy that this value is nearly 19 times lower than that of Cut6 used as positive control on the same substrate (Figure 2C).

Finally, no enzyme activity was obtained with any of the substrates for the inactive Tes $\mathrm{A}^{\mathrm{S} 104 \mathrm{~A}}$ mutant protein, where the catalytic Ser104 contained in the consensus sequence GHSMG was replaced by an Ala.

\section{Effects of Cyclipostins and Cyclophostin inhibitors $C_{y} C_{17}, C y C_{6 \beta}, C y C_{7 \beta}, C y C_{8 \beta}$ and Orlistat on TesA activity}

The CyCs are a new class of potent anti-tubercular compounds exhibiting an activity against enzymes with serine or cysteine in their active site [18, 19]. We investigated on the ability of $\mathbf{C y C}_{\mathbf{1 7}}$ to efficiently inhibit the enzyme activity of TesA to confirm this enzyme an effective target of the inhibitor. In addition, $\mathbf{C y C}_{\mathbf{7}}$ which has been found active against both extracellular and intramacrophagic $M$.

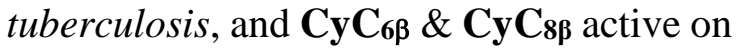
infected macrophages [18, 19], were also tested to assess their ability to inhibit TesA activity. Orlistat which has been previously reported as an inhibitor of TesA was used as positive control [33]. The chemical structures of the four CyCs analogs used in this study are provided in Figure 3.

In contrast to $\mathbf{C y}_{\mathbf{6}}, \mathbf{C y}_{7 \beta}$ and $\mathrm{CyC}_{8 \beta}$, which exhibited very weak or no inhibitory effect up to a high molar excess $x_{\mathrm{I}}=200$, a dose-dependent inhibition was observed with $\mathbf{C y C}_{17}$ and Orlistat (Figure 4A). Both compounds were found to react almost stoichiometrically with pure TesA, as confirmed by their respective inhibitor molar excess leading to $50 \%$ enzyme inhibition, i.e. $x_{\mathrm{I} 50}$ values of around 4.5 and 12.4 .
To further characterize the observed inhibitory effect, native TesA $(15 \mu \mathrm{M})$ was incubated for $30 \mathrm{~min}$ with $300 \mu \mathrm{M}$ of $\mathbf{C y C}_{\mathbf{1 7}}$ (i.e., inhibitor molar excess $x_{\mathrm{I}}=20$ ). As expected a complete loss of activity was observed when comparing the pre-treated $v s$ non-treated TesA. The resulting TesA$\mathrm{CyC}_{17}$ adduct was further incubated for $1 \mathrm{~h}$ with $10 \mu \mathrm{M}$ TAMRA-FP, a fluorescent probe known to bind to all enzymes with catalytic serine or cysteine [34]. Equal amounts of adduct were separated by SDSPAGE and visualized by Coomassie staining (Figure 4B, upper panel) or in-gel fluorescence for TAMRA detection (Figure 4B, middle panel). Pre-treatment with $\mathrm{CyC}_{17}$ (Figure 4B) resulted in a total loss of fluorescence intensity suggesting that the reaction with the TAMRA probe was strongly impaired in the TesA-CyC17 adduct. To prove the role of catalytic Ser104 in TAMRA labelling, this residue was mutated to an Ala residue. The resulting TesA ${ }^{\mathrm{S} 104 \mathrm{~A}}$ mutant was exposed to TAMRA-FP (Figure 4B, middle panel) and as expected, no fluorescence was detected confirming that the Ser104 is the catalytic nucleophile required for the binding of the probe.

The TesA-CyC17 complex was further purified on a gel filtration column for crystallization. Size exclusion chromatography coupled with online multiangle laser light scattering / quasi-elastic light scattering / refractive index (SECUV/MALS/QELS/RI) demonstrated that TesA has a molecular mass of $29 \mathrm{kDa}$ (Figure 4C - bright orange curve), which is in agreement with the theoretical molecular weight of the monomer of 29,077 Da (. In contrast, the peak of the TesA-CyC $\mathbf{C}_{17}$ complex was observed at $60 \mathrm{kDa}$ (Figure 4C - grey curve). This increase in molecular weight cannot result from binding of the $\mathbf{C y C}_{17}$ alone (Mw $\left.446 \mathrm{Da}\right)$. It is reasonable to attribute the molecular weight increase to a dimeric form of the enzyme (Figure 2). This finding suggests that some conformational changes might occur which lead to a dimerization. Such 
behavior has already been observed in several lipolytic enzymes [35] and thanks to the resolution of TesA 3D-structure a clear explanation can be proposed.

\section{Mass spectrometry analysis}

Mass spectrometry MALDI-TOF analyses were performed on TesA and Tes $\mathrm{A}^{\mathrm{S} 104 \mathrm{~A}}$ in presence or absence of $\mathbf{C y C}_{\mathbf{1 7}}$ to confirm the covalent binding of this phosphate inhibitor to the active site Ser104 residue (Figure 5). A mass increment of +316.9 Da was observed in the presence of $\mathrm{CyC}_{17}$ within the global mass of untreated TesA (Figure 5A). Conversely, no change in global mass was observed with the inactive $\mathrm{Tes}^{\mathrm{S} 104 \mathrm{~A}}$ protein, as expected (Figure 5B).

Peptide mass fingerprinting (PMF) is a useful tool to identify proteins and probe mass modifications within specific peptides [36]. This technique was used to show that TesA catalytic Ser104 covalently binds to $\mathrm{CyC}_{17}$ (Figure 5C-D), excluding any noncovalent inhibition (i.e. fixation of the inhibitor near the active site, blocking its access). Both TesA and TesA ${ }^{\text {S104A }}$ exposed to $\mathbf{C y C}_{17}$ were digested (in-gel) using trypsin to generate peptide mixtures which were then analyzed using MALDI-TOF mass spectrometry. Trypsin cuts preferentially after positively charged residues like Arg or Lys. In absence of inhibitor, tryptic digest of native TesA produced a 23 amino acid peptide harboring the catalytic serine $\left(\mathrm{I}^{94}\right.$ DDPVAFFGHS ${ }^{\mathbf{1 0 4}}$ MGGMLAFEVALR ${ }^{116}$ ) with a mass of $2480.27 \mathrm{Da}$ (Figure 5C, upper panel). Similarly, TesA ${ }^{\text {S104A }}$ produced a 2464.34 Da fragment (Figure 5D, upper panel).

There was no peptide modification observed when comparing TesA ${ }^{\mathrm{S} 104 \mathrm{~A}}$ and the Tes $\mathrm{A}^{\mathrm{S}^{104 \mathrm{~A}}-\mathbf{C y C}_{\mathbf{1 7}}}$ mixture, confirming once again the role of Ser104 in the catalytic process (Figure 5D, lower panel).

In contrast with native TesA, the peptide fragment incorporating Ser104 was absent in the TesA-CyC $\mathbf{C}$ 17 modified protein suggesting mass modification (Figure 5D, lower panel). After digestion, the Ser104 incorporating peptide is expected to be shifted by a mass increment of +316.9 Da due to the addition of the $\mathbf{C y C}_{\mathbf{1 7}}$ inhibitor. However, no isotopic peptides with the desire mass shift were detected in the digested TesA-CyC17 complex mass spectra.

It is noteworthy, however, that the observed 316.9 Da mass shift increment in global mass was 129.38 Da lower than the expected $\mathbf{C y C}_{\mathbf{1 7}}$ theoretical molecular mass of 446.28 Da (Figure 5). This size difference may arise from the specific chemical properties of phosphate inhibitor, as already described in a previous study with the Antigen 85 (Ag85) complex (i.e., Ag85A, Ag85B, and Ag85C) [37]. These secreted enzymes are responsible for the biosynthesis of trehalose dimycolate (TDM) and mycolylation of arabinogalactan, two essential lipid components of the mycobacterial cell wall $[38,39]$. In all cases, the nucleophilic attack of the catalytic serine at the phosphorus center of $\mathbf{C y C}_{\mathbf{1 7}}$ induces ring opening. The new phosphate triester thus generated can then be rapidly hydrolyzed in the presence of water molecules, resulting in the cleavage and the release of the methyl 2acetyl-4-hydroxybutyrate group (i.e., 124.1 Da), accounting for the molecular mass discrepancy observed experimentally (Figure 5E). Finally, this transiently obtained penta-coordinate phosphorus adduct may slowly rearrange to reach its stable thermodynamic state. Such mechanism is not only perfectly in line with the data generated by the proteomic experiments, but above all, it was further confirmed by the 3D structure determined below.

Overall, these data confirm the formation of a covalent complex between the TesA catalytic Ser104 and CyC17. Moreover, our results are consistent with the known and classical mechanism of action of phosphate based inhibitors [19, 37].

Crystal structure of TesA-CyC 17 
Crystallization trials of TesA alone or in the presence of phosphonate/phosphate inhibitors were performed. Only the TesA-CyC $\mathbf{1 7}$ complex led to diffracting crystals. Datasets were collected at the European Synchrotron Radiation facility (ESRF, Grenoble, France) or at SOLEIL (St Aubin, France). The structure was solved by molecular replacement using the structure of the core domain of type II thioesterase RifR from Amycolatopsis mediterranei [40] as template model (PDB id: 3FLB), and refined to $2.6 \AA$ resolution (Table $\mathbf{1}$ ). Crystals belong to space group $\mathrm{C} 222_{1}$ and contain 8 molecules per asymmetric unit. Analysis of the contacts between monomers with the PISA server [41] indicated that four groups of dimers can be identified (AC, BD, EG, FH). Moreover, the TesA dimer observed in solution might result from the hydrophobic association of the lids and active site area of two symmetry related monomers (Figure S1). This association buries an area of $1120 \AA^{2}$, amounting to $\sim 10 \%$ of the total surface $\left(11930 \AA^{2}\right)$. In the eight copies, the amino-acid chains could be traced from residues $24 / 28$ to $258 / 260$, depending upon the chain. However, two segments at positions 64 to $70 / 74$ and $155 / 158$ to $168 / 174$ could not be traced in the electron map density. A positional variation was observed between the eight copies involving the segment 135-180. In particular, the loop 135-145 exhibited two different tracks (Figure S2).

The structure of TesA consists of a core of $7 \alpha$-helices around 6 stranded parallel $\beta$-sheets, upon which protrudes a domain formed of an extended stretch followed by $2 \alpha$-helices, with connectivity $\beta 1-\alpha 1-\beta 2-\alpha 2-\beta 3-\alpha 3-\beta 4-($ ext- $\alpha 4-\alpha 5)-\beta 5-\alpha 6-$ $\beta 7-\alpha 7$ (Figure 6A). Several structurally similar folds haves been previously reported and belong to the thioesterase fold of the $\alpha / \beta$-hydrolase family [42]. TesA structure is characterized by a central parallel $\beta$-sheet and a Ser-His-Asp catalytic triad involving here Ser104, His236 and Asp208. However, the true hallmark of a $\alpha / \beta$-hydrolase are the catalytic serine loop signature $\mathrm{G}-\mathrm{X}-\mathrm{S}-\mathrm{X}-\mathrm{G}$ and its special conformation, with $\varphi$ and $\psi$ angles values of $\sim 60^{\circ}$ and $-120^{\circ}$, respectively [42]. These characteristics are indeed observed in TesA. Serine 104 is located in a loop with the sequence G-H-S-M-G and has the expected $\varphi$ and $\psi$ angles (Figure 6B). TesA was crystallized with the $\mathbf{C y C}_{\mathbf{1 7}}$ phosphonate inhibitor, which was found to be covalently bound to Ser104. The hydrocarbon tail of $\mathrm{CyC}_{17}$ could be only traced to the first 4 carbon atoms of 16 . Therest of the chain was not visible in the electron density map, probably due to disordering (Figure S3). In many complexes of lipases or esterases with phosphate, an oxygen atom of the phosphate moiety is involved in an hydrogen bond to the so-called "oxyanion hole" of the enzyme, one component being the $\mathrm{NH}$ group of the residue following the catalytic serine [43]. In the present complex, the phosphonate $\mathrm{P}-\mathrm{O}_{1}$ establishes hydrogen bonds to the main $\mathrm{NH}$ groups of Ala37 and Met105 residues (Figure 6A).

Within the $\alpha / \beta$-hydrolase family, many lipolytic enzymes have their active site controlled by a so-called (mobile) lid, formed by a surface loop [29, 35, 44, 45]. In all the cases studied, the lid domain was found to undergo a conformational change in the presence of an inhibitor, making the active site accessible to solvent [35]. In brief, the lid is a short domain that exhibits dramatic conformational changes between the apo- and the holo-forms. Since the structure has been obtained in complex with an inhibitor, we suspect that the protruding domain formed from an extended stretch of the peptide backbone, followed by two $\alpha$ helices ( $\alpha 4$ and $\alpha 5)$ might be the TesA lid in an open conformation. The TesA apo-form could not be crystallized to confirm the identification of the lid. However, we performed a DALI server search [46] to detect structurally similar proteins having possibly closed and open lids, that could be used in a comparison with TesA structure.

The DALI server returned several tens of structures with $Z>15$ and root mean 
square deviations (rmsd) lower than $3 \AA$. Among them, and of special interest, were the two crystal structures of RifR; i.e, PDB ids 3FLB ( $Z=22$; rmsd=1.2 $\AA$; used for the molecular replacement) and 3FLA ( $Z=22$; rmsd=1.2 A) [40]. When the RifR and 3FLA structures were superimposed to TesA, the core of the two enzymes coincided within $1.4 \AA$ rmsd. However, the putative lid of TesA (ext- $\alpha 4-\alpha 5)$ does not superpose to the lid of 3FLA (Figure 7A). We noticed that three helices of 3FLA do not superpose with their TesA counterparts and stack against 3fla core domain. Conversely, the domain ext- $\alpha 4-\alpha 5$ does not superpose with their 3FLA counterparts (Figure 7A). Therefore, we suggest that the ext- $\alpha 4-\alpha 5$ domain conformation is the result of a $\sim 90^{\circ}$ rotation of two helices. However, one of the helices is not structurally ordered in TesA and forms the "ext" segment (Figure 7A). The $\alpha 5$ helix results from a smaller conformational change than the one observed with some lipases like Dog and Human Gastric Lipases [47, 48]. Confirming the "lid" character of the ext$\alpha 4-\alpha 5$ domain, is the fact that the two lid helices from 3FLA cover entirely the core domain of TesA, in particular the catalytic machinery (Figure 7B). These observations convinced us that the ext- $\alpha 4-\alpha 5$ domain is a bona fide lid.

Conversely, we scanned the DALI server hits to find a structure well superposed with TesA lid. The recently determined structure of the thioesterase domain of $M$. tuberculosis Polyketide Synthase 13 (Pks13; PDB id: 5V3X; Z= 20; rmsd=2.2 A) [49] returned as an excellent hit by DALI. This structure is in complex with an inhibitor (TAM1), and indeed, the superposition with TesA shows that both lid domains coincide well. In addition, the Pks13 thioesterase inhibitor TAM1 is located close to $\mathbf{C y C 1 7}$ (Figure 7A). However, a Pks13 thioesterase extra domain of two $\alpha$-helices does not exist in TesA (Figure $\mathbf{8 A}$ and $\mathbf{B}$ ). TAM1 is sandwiched between these two $\alpha$-helices and the lid, resulting in a catalytic crevice of
Pks13 thioesterase being much more restricted and deeper than that of TesA (Figure 8A and B).

\section{Discussion}

Although M. tuberculosis, the main cause of human TB, can be traced back to 70,000 years ago [50], it currently remains the ninth cause of death worldwide and represents the primary cause of death by single infectious pathogen, even ranking above HIV/AIDS [1]. The difficulties in treating the disease mainly result from the fact that $M$. tuberculosis encounters diverse microenvironments and can be found in a variety of metabolic states during the infection of the human host [51]. Available antituberculosis drugs cannot act against all metabolic states of the bacteria, e.g. replicating vs non-replicating $M$. tuberculosis, or against multi- and extensively-drug resistant $M$. tuberculosis (M/XDR-TB) vs. classical strain. Moreover, despite the use of promising new drugs; such as bedaquiline which inhibits ATP synthase, and the nitroimidazoles delamanid and pretomanid which inhibit mycolic acid synthesis as well as energy production [52, 53]; there is an urgent need to discover new drugs to fight several mycobacterial infections such as Tuberculosis, Leprosis and Buruli Ulcer. The majority of drugs commercially available target a single molecule/process involved in the synthesis pathways essential for the bacterial survival during the infection process. Unfortunately, impairing only one protein may lead to the rapid emergence of resistant strains. Consequently, to overcome these challenges, TB treatment is a 6-month regimen of antibiotics: isoniazid, rifampicin, ethambutol and pyrazinamide. This treatment regimen is often extended to 9-12 months to treat difficult cases. The inherent difficulty to be compliant with such a long treatment is in part responsible for the appearance of resistant strains, representing a new challenge to achieve 
control of the disease. As reported by Zumla et al. in 2013 "there is growing awareness of the need for drugs that can kill M. tuberculosis in its different physiological states" [54]. The priority for new drug development against TB should thus be focused on the discovery of new candidates that would be able to i) reduce the treatment duration and cost; ii) impact several target enzymes; and iii) act on several physiological states of the bacteria.

In this context, we have demonstrated that the $\mathbf{C y C}$ analogs represent powerful and selective inhibitors of mycobacterial enzymes [14, 15], with no effect on the mammalian enzymes initially targeted by natural parent molecules [1417]. The selectivity of the $\mathbf{C y C}$ derivatives toward the mycobacterial but not the human enzymes, is therefore highly valuable and prompted us to consider them as potential anti-tubercular agents. Accordingly, among the set of $27 \mathbf{C y C}$ analogs previously evaluated against $M$. tuberculosis H37Rv, eight compounds were able to efficiently inhibit M. tuberculosis growth either extracellularly or within macrophages at similar concentrations as isoniazid and ethambutol [19]. Importantly, the best CyC compounds showed absence of toxicity in mammalian cells at concentrations up to $100 \mu \mathrm{M}$ [19]. More particularly, СуC 6 , $\mathrm{CyC}_{7 \beta}$ and $\mathrm{CyC}_{8 \beta}$ were mainly active against intracellularly-replicating mycobacteria (MIC 50 of 12.6, 3.1 and 11.7 $\mu \mathrm{M}$, respectively). In contrast, $\mathbf{C y C}_{17}$ was a potent inhibitor of in vitro growth $\left(\mathrm{MIC}_{50} \sim\right.$ $0.5 \mu \mathrm{M}$ ) but failed to show activity against intracellular bacilli. These findings suggest that there are several modes of action of these related compounds (extracellular vs. intracellular) and that they probably target several enzymes. Using an activity-based protein profiling (ABPP) approach, 23 putative protein targets for $\mathbf{C y C}_{\mathbf{1 7}}$ were identified, including the thioesterase TesA [19].

Overall, these first results support the assumption that $\mathbf{C y C}$ inhibitors are multi-target compounds leading to the inhibition of $M$. tuberculosis growth through the inhibition of various mycobacterial Ser- or Cys-containing enzymes involved in important physiological processes. In addition, the lack of cytotoxicity towards host cells [19], makes these $\mathbf{C y C}$ analogs exciting molecules to be further evaluated as new lead candidates for treating the disease.

Herein we provide clear evidence that TesA is strongly inhibited by $\mathbf{C y C}_{\mathbf{1 7}}$ but not by $\mathrm{CyC}_{6 \beta}, \mathrm{CyC}_{7 \beta}$ or $\mathrm{CyC}_{8 \beta}$ (Figure 3A). As showed in the crystal structure of TesA in complex with the $\mathbf{C y} \mathbf{C}_{\mathbf{1 7}}$, the enzyme inhibition results from the phosphorylation of the catalytic Ser104 (Figure 6A). The inhibitory effect of $\mathrm{CyC}_{17}$ on TesA allows the validation, a posteriori, of this enzyme as an effective target of this molecule, as initially postponed via previous ABPP experiments [19].

However, the absence of in vitro inhibition of TesA by $\mathbf{C y C}_{6 \boldsymbol{\beta}}, \mathbf{C y C}_{7 \boldsymbol{\beta}}$ or $\mathrm{CyC}_{\mathbf{3} \beta}$ strengthens the fact that $M$. tuberculosis bacterial clearance inside infected macrophages, observed during ex vivo experiments in presence of these molecules [19], does not result from the inhibition of this enzyme. In contrast the Antigen 85 complex is targeted by $\mathbf{C y C}_{7 \boldsymbol{\beta}}$, $\mathbf{C y C}_{8 \beta}$ as well as $\mathbf{C y C}_{17}$ [37]. From a structure-activity relationship (SAR) point of view, $\mathbf{C y C}_{6 \beta}, \mathbf{C y C}_{7 \beta}$ and $\mathbf{C y C}_{8 \beta}$ are phosphonate analogs while $\mathbf{C y C}_{17}$ is a phosphate (Figure 3). However, such a chemical change (i.e., phosphonate vs. phosphate) is not a sufficient criteria to explain the observed difference in their inhibitory potency, since any catalytic serine/cysteine is able to react similarly with both chemical species $[14,19]$. A more plausible explanation relies on the core structure of these compounds and more precisely on the positioning of the alkyl chain. If phosphonates $\mathbf{C y}_{6 \beta} \mathbf{8} \boldsymbol{\beta}$ are best described by the relationship between the OMe on phosphorus and the $\mathrm{H}$-substituent on the C-5 carbon atom, being here in a cis ( $\beta$-isomer) conformation; with $\mathbf{C y C}_{\mathbf{1 7}}$, the lipophilic C16-alkyl chain is directly 
carried by the phosphorus atom. Accordingly and as reported previously [14], the presence of such lipophilic alkyl chain on the $\gamma$-carbon of the enolphosphonate ring may significantly affect and modify the biological activity of the corresponding monocyclic analogs. The exquisite chemoselectivity exerted by TesA for $\mathbf{C y C}_{17}$ phosphate over the phosphonates could result from a steric hindrance provided by lateral alkyl chain located at the C5-carbone atom in $\mathbf{C y C}_{\boldsymbol{\alpha} \beta}$-8 $\beta$ phosphonates making reaction between the phosphorus atom with the catalytic Ser104 extremely difficult, as already observed with mammalian lipases [14].

The biochemical and mass spectrometry experiments involving $\mathbf{C y C}_{\mathbf{1 7}}$, coupled with site directed mutagenesis conducted on Ser104 have confirmed the covalent binding of $\mathbf{C y C}_{\mathbf{1}}$ to the catalytic serine of TesA. Remarkably, after phosphorylation of the serine by $\mathbf{C y C}_{\mathbf{1 7}}$, a rearrangement of the inner structure of the bound inhibitor occurs with the release of methyl 2-acetyl 4-hydroxybutyrate group (Figure 5E). Such chemical rearrangement that has been previously observed in the inhibited Ag85C-CyC17 complex [37], and can therefore be considered as a signature of the $\mathbf{C y C}_{\mathbf{1 7}}$ reactivity with Ser- and Cyscontaining enzymes.

As found in some other thioesterases and many lipolytic enzymes, TesA 3D structure reveals the presence of a lid protecting the catalytic site and composed by $2 \alpha$-helices $[40,49,55]$. These structural observations are in line with the results of $p N P$ ester hydrolysis showing a clear increase in TesA activity in presence of surfactant (i.e., Triton X-100 - Figure 2B). In the structure depicted in Figure 6, the lid is in open conformation allowing the interaction between $\mathbf{C y C}_{\mathbf{1 7}}$ and the active Ser104. Moreover, in this crystal structure the polar head of $\mathbf{C y C} \mathbf{1 7}$ is also clearly covalently bound to Ser104 and its hydrocarbon tail seems to be floating towards the hydrophobic crevice located between the core and the lid. Only a few of the carbons close to the phosphate group could be modelled in the electron density map (Figure S2). Despite many attempts, no suitable crystals were obtained in absence of inhibitor suggesting that TesA is difficult to crystallize in closed conformation. The presence of the inhibitor could, however promote the lid opening which results in the formation of dimers (Figure 4C) that allows good quality of crystals to form.

In the present study, we bring new evidence that the $\mathbf{C y C}$ analogs impair various target enzymes with different activities. Consequently, a way to enhance the efficiency of this new family of antitubercular molecules would be the use of these $\mathbf{C y C}$ as a cocktail mixture, or together with known antibiotics to treat intra and/or extracellular bacteria.

\section{Material and Methods}

\section{Bacterial strains and growth condition}

Escherichia coli DH10 $\beta$ cells (Invitrogen) used in cloning experiments were grown at $37^{\circ} \mathrm{C}$ in Luria Bertani (LB) or on LB agar plates. In the expression experiments, Escherichia coli T7 Iq pLysS strain was used to carry the expression vector and express protein. Terrific Broth (TB) broth (Difco) was used to grow the strain with shaking at $250 \mathrm{rpm}$ and $37^{\circ} \mathrm{C}$. All the media were supplemented with 100 $\mu \mathrm{g} / \mathrm{mL}$ ampicillin.

\section{Cloning, expression and purification of proteins}

From genomic DNA of $M$. tuberculosis $\mathrm{H} 37 \mathrm{Rv}$, full-length of $R v 2928$ gene (786 pb) encoding TesA enzyme (261 AA, MW 29,077 Da, pI 5.11) was amplified using the Phusion ${ }^{\circledR}$ DNA polymerase (ThermoFisher Scientific). After purification the PCR product was cloned into the Gateway pDEST14 expression vector using Gateway cloning technology as described previously. The forward primers used is TesA-Fwd ${ }^{5}$ GGGG ACA AGT TTG TAC AAA AAA GCA GGC TTC GAA GGA GAT AGA ACC ATG 
CAT CAC CAT CAC CAT CAC GAA AAC CTG TAC TTC CAG GGT CTG GCC CGT CAC GGA CCA CGC TAT G ${ }^{3}$, and the reverse primer is TesA-Rev ${ }^{5}$ CTA AGC TCG ATC ATG CCA TTG GAG TG ${ }^{3}$. The final construct encodes the TesA gene fused to a N-terminal hexahistidine tag followed by a TEV cleavage site (bold). After checking the integrity of the DNA sequence by DNA sequencing (GATC-BIOTECH, Germany), the recombinant vector was transformed into Escherichia coli T7 Iq pLysS cells (New England Biolabs) and then expressed and purified. Briefly, the cells were grown in Terrific Broth (TB) at $37^{\circ} \mathrm{C}$ until $\mathrm{OD}_{600 \mathrm{~nm}}$ reached 0.6-1.0. Expression of TesA was induced by $0.5 \mathrm{mM}$ isopropyl- $\beta$-thiogalactoside (IPTG) for overnight at $17^{\circ} \mathrm{C}$. Cells were harvested by centrifugation at $4,000 \times g$ for $15 \mathrm{~min}$ at $4^{\circ} \mathrm{C}$, resuspended in lysis buffer $(50 \mathrm{mM}$ Tris-HCl, $\mathrm{pH} 8,300$ $\mathrm{mM} \mathrm{NaCl}, 10 \mathrm{mM}$ imidazole, $0.25 \mathrm{mg} / \mathrm{mL}$ lysozyme) and lysed by sonication at $50 \%$ power during $4 \mathrm{~min}$, with 45 short burst of $45 \mathrm{~s}$ followed by intervals of $15 \mathrm{~s}$ for cooling. The lysate was cleared by centrifugation at $18,000 \times g$ for 45 minutes and the supernatant was loaded onto a $\mathrm{Ni}^{2+}$ affinity chromatography column (HisTrap 5 $\mathrm{mL}$, GE Healthcare). The protein was washed with buffer A (20 mM Tris- $\mathrm{HCl}, \mathrm{pH}$ $8,150 \mathrm{mM} \mathrm{NaCl}$ ) containing $50 \mathrm{mM}$ imidazole and eluted with buffer $\mathrm{A}$ containing $250 \mathrm{mM}$ imidazole. The hexahistidine tag was cleaved by incubation of the protein with the histidine-tagged TEV protease (ratio 40:1, protein: TEV, $w / w$ ) overnight, coupled with dialysis against buffer A to eliminate imidazole. The untagged TesA was obtained in the flow through of a second $\mathrm{Ni}^{2+}$ affinity chromatography and further purified by preparative Superdex 75 (GE Healthcare) gel filtration in buffer A. TesA was concentrated to $15 \mathrm{mg} / \mathrm{mL}$ for crystallization studies and other experiments. Complexes of TesA with different $\mathbf{C y C}$ inhibitors $\left(\mathbf{C y C}_{17}, \mathbf{C y C}_{\mathbf{7}}\right.$, $\mathbf{C y C}_{8 \beta}$ ) were prepared by mixing pure TesA with each $\mathbf{C y C}$, previously dissolved in DMSO, at a molar excess $\left(x_{\mathrm{I}}\right)$ of 100 . The mixture was left at $4{ }^{\circ} \mathrm{C}$ for at least $1 \mathrm{~h}$ before submitted to an additional preparative Superdex 75 gel filtration in buffer A. TesA-inhibitor complexes were concentrated to $15 \mathrm{mg} / \mathrm{mL}$ for further crystallization studies.

\section{TesA mutant construction}

The pDEST14-TesA ${ }^{\text {S104A }}$ mutant plasmid was constructed by the QuickChange method using pDEST14TesA as matrix, the Phusion ${ }^{\circledR}$ DNA polymerase (ThermoFisher Scientific), the forward primer TesA ${ }^{\text {S104A-Fwd }}{ }^{5}$ G GTG GCA TTC TTT GGG CAC GCT ATG GGC GGA ATG CTA GCC TTC ${ }^{3}$, , and the reverse primer TesA ${ }^{\text {S104A }}-$ Rev $^{5}{ }^{\prime}$ GAA GGC TAG CAT TCC GCC CAT AGC GTG CCC AAA GAA TGC CAC C ${ }^{3}$ ' (Ser-to-Ala mutation in bold). After DNA sequencing, the recombinant plasmid was transformed into Escherichia coli T7 Iq pLysS cells and the recombinant enzyme was produced and purified as described above.

\section{MALS/QELS/UV/RI-coupled size exclusion chromatography}

Size exclusion chromatography was performed on an Alliance 2695 HPLC system (Waters) using a precalibrated KW802.5 column (Shodex) run in $20 \mathrm{mM}$ Tris- $\mathrm{HCl}, \mathrm{pH} 8,150 \mathrm{mM} \mathrm{NaCl}$ at 0.5 $\mathrm{mL} / \mathrm{min}$. MALS, UV spectrophotometry, QELS and RI were achieved with MiniDawn Treos (Wyatt Technology), a Photo Diode Array 2996 (Waters), a DynaPro (Wyatt Technology) and an Optilab rEX (Wyatt Technology), respectively, as described [56]. Mass and hydrodynamic radius calculation was done with ASTRA software (Wyatt Technology) using a dn/dc value of $0.185 \mathrm{~mL} / \mathrm{g}$.

\section{Enzymatic activity}

\section{Esterase activity}

The esterase enzyme activity was performed as previously described with minor modifications [57]. In brief, paranitrophenyl $(p N P)$ esters (Sigma-Aldrich, 
Saint-Quentin Fallavier, France) with different carbon chain lengths were used as substrates, including $p \mathrm{NP}$ acetate ( $p \mathrm{NP}$ $\mathrm{C} 2)$, valerate ( $p$ NP-C5), caprylate ( $p$ NP$\mathrm{C} 8$ ), laurate $(p \mathrm{NP}-\mathrm{C} 12)$, myristate ( $p \mathrm{NP}-$ C14) palmitate ( $p$ NP-C16) and stearate ( $p$ NP-C18). Stock solution (from 20 to 100 $\mathrm{mM}$ ) of each substrate was freshly prepared in acetonitrile. Release of $p \mathrm{NP}$ was monitored at $410 \mathrm{~nm}$ and $\mathrm{pH} 7.5$ using a 96well plate spectrophotometer (PowerWave $^{\mathrm{TM}}$, Bio-Tek Instruments) and quantified using a $p$ NP calibration curve (10 $\mu \mathrm{M}$ to $0.5 \mathrm{mM})$ with apparent $\varepsilon_{(\lambda=410 \mathrm{~nm})}=$ $8.4 \mathrm{mM}^{-1}$ and $6.0 \mathrm{mM}^{-1}$ when using $0.5 \%$ $(w / v)$ Triton X-100. Enzymatic reactions were performed at $37{ }^{\circ} \mathrm{C}$ over a period of 20 min in a 96-well microplate filled with 100 $\mathrm{mM}$ Tris- $\mathrm{HCl}$ buffer ( $\mathrm{pH}$ 7.5) containing $100 \mathrm{mM} \mathrm{NaCl}$ to a final volume of $200 \mu \mathrm{L}$. In each well, $2 \mathrm{mM}$ of substrate and $50 \mu \mathrm{g}$ of enzyme $(8.6 \mu \mathrm{M}$ final concentration) were added. For each $p$ NP ester, the assay was performed in presence or absence of $0.5 \%(w / v)$ Triton $\mathrm{X}-100$ in the buffer. When Triton $\mathrm{X}-100$ was present, the $p \mathrm{NP}$ esters were first solubilized in the buffer by sonication in a water bath for $1 \mathrm{~min}$. Activities were expressed in international units (U), corresponding to $1 \mu \mathrm{mol}$ of $p \mathrm{NP}$ released per min. Specific activities were expressed as $\mathrm{mU} / \mathrm{mg}$ of pure enzyme. The same conditions were also applied to the inactive $\mathrm{Tes}^{\mathrm{S} 104 \mathrm{~A}}$ catalytic mutant. Negative controls included denaturated enzyme (10 min boiling) and protein filtrates (obtained during concentration steps). All experiments were performed at least in triplicate.

\section{Thioesterase activity assay}

Hydrolysis of Palmitoyl-CoEnzyme A (Palmitoyl-CoA) (Sigma-Aldrich) was used to measure thioesterase activity. After hydrolysis of the thioester bond, the free sulfur on CoA is attacked by DTNB (5-5'Dithio-bis (2-nitrobenzoic acid)) (SigmaAldrich) which releases a measurable nitrophenyl group, $\mathrm{TNB}^{2-}$ (5-Thio-2nitrobenzoate) ion detectable at $415 \mathrm{~nm}$. Fresh stock solution of Palmitoyl-CoA (1
$\mathrm{mM})$ and DTNB $(0.4 \mathrm{mM})$ were prepared in water. Palmitoyl-CoA and DTNB were added in each well of a 96-well microplate at final concentration of $10 \mu \mathrm{M}$. The thioesterase activity of TesA $(23.5 \mu \mathrm{g}-4.0$ $\mu \mathrm{M}$ final concentration) was compared to that of Cut6 (Rv3802c) $(7.5 \mu \mathrm{g}-1.1 \mu \mathrm{M}$ final concentration) [28] which was used as positive control. Triplicate assays were done at $\mathrm{pH} 8.0$ in $10 \mathrm{mM}$ Tris, $300 \mathrm{mM}$ $\mathrm{NaCl}$ buffer. The absorbance at $415 \mathrm{~nm}$ was continuously measured and the release of $\mathrm{TNB}^{2-}$ was quantified using a calibration curve.

\section{Inhibition by $\mathrm{CyC17}, \mathrm{CyC} 7 \beta, \mathrm{CyC} \beta \beta$ and Orlistat}

Inhibition experiments were carried out using a classic lipase inhibitor preincubation method, as previously described $[14,15]$. Mother solution of each inhibitor $\mathrm{CyC}_{17}, \mathrm{CyC}_{6 \beta}, \mathrm{CyC}_{7 \beta}, \mathrm{CyC}_{8 \beta}$ and Orlistat were prepared in DMSO at a concentration of $4.6 \mathrm{mg} / \mathrm{mL}, 5.7 \mathrm{mg} / \mathrm{mL}, 5.9 \mathrm{mg} / \mathrm{mL}$ and $5.0 \mathrm{mg} / \mathrm{mL}$, respectively. TesA was preincubated for $30 \mathrm{~min}$ at $37^{\circ} \mathrm{C}$ with each $\mathbf{C y C}$ at various inhibitor molar excess $\left(x_{\mathrm{I}}\right)$ ranging from 1 to 100 related to $1 \mathrm{~mol}$ of enzyme. In each case, control experiments were performed with the same volume of solvent, without inhibitor. Residual activities were assayed using the colorimetric assays with the substrate $p$ NP$\mathrm{C} 8$ as described above. The variation in the residual enzyme activity allowed determination of the inhibitor molar excess which reduced the enzyme activity to $50 \%$ of its initial value $\left(x_{150}\right)$. Results are expressed as mean values of at least two independent assays (CV\%, 5.0\%).

In view of the specific mechanism of action of lipolytic enzymes [35], the Michaelis-Menten-Henri model no longer applies [58] and the $K_{\mathrm{m}}, K_{\mathrm{i}}$ and $\mathrm{IC}_{50}$ values often estimated for lipolytic enzymes and expressed in terms of volume concentrations are irrelevant when insoluble substrates and/or inhibitors are involved, or when conformational changes related to lid opening are occurring [29]. 
The use of more appropriate kinetic constants such as the inhibitor molar excess leading to $50 \%$ enzyme inhibition (i.e., $x_{150}$ value) $[14,15,59]$ is therefore recommended when assessing the inhibitory potency of insoluble inhibitors, such as these cyclic enol-phosphorus derivatives. Thereby, a $x_{150}$ value of 0.5 is synonymous with a $1: 1$ stoichiometric ratio between the inhibitor and the lipolytic enzyme, and is therefore the highest level of inhibitory activity that can be achieved.

\section{Mass spectrometry analyses}

\section{Total mass analyses}

Total mass analyses were performed on a MALDI-TOF-TOF Bruker Ultraflex III spectrometer (Bruker Daltonics, Wissembourg, France) controlled by the Flexcontrol 3.0 package (Build 51). This instrument was used at a maximum accelerating potential of $25 \mathrm{kV}$ and was operated in linear mode using the $\mathrm{m} / \mathrm{z}$ range from 20,000 to 100,000 (LP_66 KDa Method). Five external standards (Protein Calibration Standard II, Bruker Daltonics) were used to calibrate each spectrum to a mass accuracy within $200 \mathrm{ppm}$. Peak picking was performed with Flexanalysis 3.0 software (Bruker) with an adapted analysis method. To eliminate salts from the samples solutions, $10 \mu \mathrm{L}$ of each preparation was submitted to a desalting step on a C4 Zip-Tip $\mu$ column (Millipore). One $\mu \mathrm{L}$ of desalted sample was mixed with $1 \mu \mathrm{L}$ sinapinic acid matrix in a $50 \%$ acetonitrile $/ 0.3 \%$ trifluoroacetic acid (TFA) mixture $(1: 1, v / v)$. Then $1 \mu \mathrm{L}$ was spotted on the target, dried and analysed with the LP_66Kda method. Peak picking was performed with Flexanalysis 3.0 software (Bruker) with an adapted analysis method. Parameters used were as follows: SNAP peak detection algorithm, $\mathrm{S} / \mathrm{N}$ threshold fixed to 6 and a quality factor threshold of 30.

\section{Tryptic digestions and peptide mass analyses}

Protein denaturating SDS-PAGE was performed on Nu-Page 4-12\% Bis-Tris gels (Invitrogen, Life Technologies, Carlsbad, CA). The gel was stained with Coomassie blue (Imperial Protein stain, Pierce). Protein spots $(\sim 10 \mu \mathrm{g})$ excised from Coomassie blue stained gels were subjected to in-gel digestion with trypsin (2 $\mu \mathrm{L}-12.5 \mathrm{ng} / \mu \mathrm{L}$ ) (Sequencing grade modified porcine trypsin; Promega, Madison, WI, USA) according to a modified protocol from Shevchenko et al. [60]. Tryptic peptides were then extracted from the gel by successive treatment with $5 \%$ formic acid and $60 \%$ acetonitrile $/ 5 \%$ formic acid, each treatment followed by a $10 \mathrm{~min}$ sonication. Extracts were pooled and dried in a Speedvac evaporator.

Peptide mass analyses were performed on a MALDI-TOF-TOF Bruker (as described above) operating in reflectron mode using the $\mathrm{m} / \mathrm{z}$ range from 600 to 3700 (RP Proteomics_2017 Method). The laser frequency was fixed to $200 \mathrm{~Hz}$ and approximately 500 shots by sample were cumulated. Five external standards (Peptide Calibration Standard, Bruker Daltonics) were used to calibrate each spectrum to a mass accuracy within $50 \mathrm{ppm}$. One $\mu \mathrm{L}$ of sample was mixed with $1 \mu \mathrm{L}$ of a saturated HCCA ( $\alpha$-cyano-4-hydroxycinnamic acid) solution in acetonitrile $/ 0.3 \%$ TFA $(1: 1, v / v)$. Then $1 \mu \mathrm{L}$ was spotted on the target, dried and analysed with the RP Proteomics 2015 method. Peak picking was performed with Flexanalysis 3.0 software (Bruker), using the following parameters: SNAP peak detection algorithm, $\mathrm{S} / \mathrm{N}$ threshold fixed to 6 and a quality factor threshold of 30 .

\section{Crystallization and data processing of TesA}

Crystallization trials were carried out using the sitting-drop vapour diffusion method [61]. The reservoirs of the Greiner plates were filled with a TECAN pipetting robot, and nanodrops were dispensed by a Mosquito robot (TTP Labtech). No crystal hits were found when using apo-form of TesA, although many different screening conditions and protein concentrations were used. All three complexes of inhibitor- 
bound TesA (TesA-CyC17, TesA-CyC $\mathbf{C}_{\mathbf{7}}$, TesA-CyC $8 \beta$, at a molar excess $\left(x_{\mathrm{I}}\right)$ of 100$)$ resulted in different crystal forms, however only crystals of TesA-CyC $\mathbf{C} \mathbf{1 7}$ appeared in 45\% PEG 600, 0.1 M HEPES pH 7.5 diffracted well. The resolution of these crystals was improved by adding of $10 \%$ $(v / v)$ 2-methyl-2,4-pentanediol (MPD) and $5 \mathrm{mM} \beta$-Octyl glucoside into the well solution before drop preparation. Crystals were directly cryo-cooled in well solution without any cryo-protection. Datasets were collected at the SOLEIL synchrotron (St Aubin, France). Data were processed with XDSME [62] and the structure was solved by molecular replacement with the structure of the core domain of RifR a thioesterase from the rifamycin biosynthetic pathway from Amycolatopsis mediterranei as search model (PDB id: 3FLB) [40] and using Phaser from the PHENIX software suite [63].

Crystal form 1 had the F222 space group with unit cell parameters: $\mathrm{a}=77.58$, $\mathrm{b}=224.65, \mathrm{c}=226.62 \AA$ and $\alpha=\beta=\gamma=90^{\circ}$, 4 molecule of TesA in 1 asymmetric unit. Crystal form 2 had the $\mathrm{C} 222{ }_{1}$ space group with unit cell parameters: $a=79.07$, $\mathrm{b}=224.58, \mathrm{c}=222.21 \AA$ and $\alpha=\beta=\gamma=90^{\circ}$, 8 molecules of TesA in 1 asymmetric unit. Manual adjustments of the model were performed with Coot [64] and the structure was refined to $2.75 \AA$ for crystal form 1 and 2.6 A for crystal form 2 with BUSTER [65]. Covalent link between $\mathbf{C y C}_{17}$ and catalytic serine and restraints for the inhibitor were generated by Jligand [66]. Data collection and refinement statistics are displayed in Table 1.

\section{Funding/Acknowledgments}

This work was supported by the CNRS and Aix Marseille University. P.C.N was supported by the $\mathrm{PhD}$ Training program from the University of Science and Technology of Hanoi (837267E). V.S.N was supported by a $\mathrm{PhD}$ grant from the French Embassy in Vietnam (792803C). Proteomics analyses were supported by the Institut Paoli-Calmettes and the Centre de Recherche en Cancérologie de Marseille.
Proteomic analyses were done using the mass spectrometry facility of Marseille Proteomics (marseille-proteomique.univamu.fr) supported by IBISA (Infrastructures Biologie Santé et Agronomie), the Cancéropôle PACA, the Provence-Alpes-Côte d'Azur Region, the Institut Paoli-Calmettes and the Centre de Recherche en Cancérologie de Marseille. The atomic coordinates and structure factors (codes 6FW5 and 6FVJ) have been deposited in the Protein Data Bank (http://wwpdb.org/).

\section{References}

[1] WHO. http://www.who.int/tb/publications/global report/en/. 2017.

[2] Hett EC, Rubin EJ. Bacterial growth and cell division: a mycobacterial perspective. Microbiol Mol Biol Rev. 2008;72:126-56, table of contents.

[3] Jackson M. The Mycobacterial Cell Envelope-Lipids. Cold Spring Harb Perspect Med. 2014;4.

[4] Camacho LR, Constant P, Raynaud C, Lanéelle M-A, Triccas JA, Gicquel B, et al. Analysis of the Phthiocerol Dimycocerosate Locus ofMycobacterium tuberculosis : evidence that this lipid is involved in the cell wall permeability barrier. J Biol Chem. 2001;276:19845-54.

[5] Constant P, Perez E, Malaga W, Laneelle MA, Saurel O, Daffe M, et al. Role of the pks15/1 gene in the biosynthesis of phenolglycolipids in the Mycobacterium tuberculosis complex. Evidence that all strains synthesize glycosylated phydroxybenzoic methyl esters and that strains devoid of phenolglycolipids harbor a frameshift mutation in the pks15/1 gene. J Biol Chem. 2002;277:38148-58.

[6] Daffé M, Lacave C, Laneelle MA, Laneelle G. Structure of the major triglycosyl phenol-phthiocerol of Mycobacterium tuberculosis (strain Canetti). Eur J Biochem. 1987;167:155-60. [7] Huet G, Constant P, Malaga W, Laneelle MA, Kremer K, van Soolingen D, et al. A lipid profile typifies the Beijing strains of 
Mycobacterium tuberculosis: identification of a mutation responsible for a modification of the structures of phthiocerol dimycocerosates and phenolic glycolipids. J Biol Chem. 2009;284:27101-13.

[8] Reed MB, Domenech P, Manca C, Su H, Barczak AK, Kreiswirth BN, et al. A glycolipid of hypervirulent tuberculosis strains that inhibits the innate immune response. Nature. 2004;431:84-7.

[9] Mohandas P, Budell WC, Mueller E, Au A, Bythrow GV, Quadri LE. Pleiotropic consequences of gene knockouts in the phthiocerol dimycocerosate and phenolic glycolipid biosynthetic gene cluster of the opportunistic human pathogen Mycobacterium marinum. FEMS Microbiol Lett. 2016;363:fnw016.

[10] Alibaud L, Rombouts Y, Trivelli X, Burguiere A, Cirillo SL, Cirillo JD, et al. A Mycobacterium marinum TesA mutant defective for major cell wall-associated lipids is highly attenuated in Dictyostelium discoideum and zebrafish embryos. Mol Microbiol. 2011;80:919-34.

[11] Astarie-Dequeker C, Le Guyader L, Malaga W, Seaphanh FK, Chalut C, Lopez A, et al. Phthiocerol dimycocerosates of $\mathrm{M}$. tuberculosis participate in macrophage invasion by inducing changes in the organization of plasma membrane lipids. PLoS Pathog. 2009;5:e1000289.

[12] Dhungel S, Ranjit C, Sapkota BR, Macdonald M. Role of PGL-I of M. leprae in TNF-alpha production by in vitro whole blood assay. Nepal Med Coll J. 2008;10:13.

[13] Ferreras JA, Stirrett KL, Lu X, Ryu JS, Soll CE, Tan DS, et al. Mycobacterial phenolic glycolipid virulence factor biosynthesis: mechanism and smallmolecule inhibition of polyketide chain initiation. Chem Biol. 2008;15:51-61.

[14] Point V, Malla RK, Diomande S, Martin BP, Delorme V, Carriere F, et al. Synthesis and kinetic evaluation of cyclophostin and cyclipostins phosphonate analogs as selective and potent inhibitors of microbial lipases. J Med Chem. 2012;55:10204-19.
[15] Point V, Malla RK, Carriere F, Canaan S, Spilling CD, Cavalier JF. Enantioselective inhibition of microbial lipolytic enzymes by nonracemic monocyclic enolphosphonate analogues of cyclophostin. J Med Chem. 2013;56:4393401.

[16] Martin BP, Vasilieva E, Dupureur CM, Spilling CD. Synthesis and comparison of the biological activity of monocyclic phosphonate, difluorophosphonate and phosphate analogs of the natural $\mathrm{AChE}$ inhibitor cyclophostin. Bioorg Med Chem. 2015;23:7529-34.

[17] Vasilieva E, Dutta S, Malla RK, Martin BP, Spilling CD, Dupureur CM. Rat hormone sensitive lipase inhibition by cyclipostins and their analogs. Bioorg Med Chem. 2015;23:944-52.

[18] Nguyen PC, Madani A, Santucci P, Martin BP, Paudel RR, Delattre S, et al. Cyclophostin and cyclipostins analogs, new promising molecules to treat mycobacterialrelated diseases. Int $\mathbf{J}$ Antimicrob Agents. 2018;51:651-4.

[19] Nguyen PC, Delorme V, Benarouche A, Martin BP, Paudel R, Gnawali GR, et al. Cyclipostins and Cyclophostin analogs as promising compounds in the fight against tuberculosis. Sci Rep. 2017;7:11751.

[20] Chavadi SS, Edupuganti UR, Vergnolle O, Fatima I, Singh SM, Soll CE, et al. Inactivation of tesA reduces cell wall lipid production and increases drug susceptibility in mycobacteria. $\mathrm{J}$ Biol Chem. 2011;286:24616-25.

[21] de Souza GA, Wiker HG. A proteomic view of mycobacteria. Proteomics. 2011;11:3118-27.

[22] Gu S, Chen J, Dobos KM, Bradbury EM, Belisle JT, Chen X. Comprehensive proteomic profiling of the membrane constituents of a Mycobacterium tuberculosis strain. Mol Cell Proteomics. 2003;2:1284-96.

[23] Griffin JE, Gawronski JD, Dejesus MA, Ioerger TR, Akerley BJ, Sassetti CM. High-resolution phenotypic profiling defines genes essential for mycobacterial 
growth and cholesterol catabolism. PLoS Pathog. 2011;7:e1002251.

[24] Waddell SJ, Chung GA, Gibson KJ, Everett MJ, Minnikin DE, Besra GS, et al. Inactivation of polyketide synthase and related genes results in the loss of complex lipids in Mycobacterium tuberculosis H37Rv. Lett Appl Microbiol. 2005;40:2016.

[25] Rao A, Ranganathan A. Interaction studies on proteins encoded by the phthiocerol dimycocerosate locus of Mycobacterium tuberculosis. Mol Genet Genomics. 2004;272:571-9.

[26] Kovacic F, Granzin J, Wilhelm S, Kojic-Prodic B, Batra-Safferling R, Jaeger KE. Structural and functional characterisation of TesA - a novel lysophospholipase A from Pseudomonas aeruginosa. PLoS One. 2013;8:e69125.

[27] Crellin PK, Vivian JP, Scoble J, Chow FM, West NP, Brammananth R, et al. Tetrahydrolipstatin inhibition, functional analyses, and three-dimensional structure of a lipase essential for mycobacterial viability. J Biol Chem. 2010;285:30050-60. [28] Parker SK, Barkley RM, Rino JG, Vasil ML. Mycobacterium tuberculosis Rv3802c encodes a phospholipase/thioesterase and is inhibited by the antimycobacterial agent tetrahydrolipstatin. PLoS One. 2009;4:e4281.

[29] Delorme V, Dhouib R, Canaan S, Fotiadu F, Carrière F, Cavalier J-F. Effects of Surfactants on Lipase Structure, Activity and Inhibition. Pharmaceutical Research. 2011;28:1831-42.

[30] Chahinian H, Nini L, Boitard E, Dubes JP, Comeau LC, Sarda L. Distinction between esterases and lipases: a kinetic study with vinyl esters and TAG. Lipids. 2002;37:653-62.

[31] Ulker S, Placidi C, Point V, Gadenne B, Serveau-Avesque C, Canaan S, et al. New lipase assay using Pomegranate oil coating in microtiter plates. Biochimie. 2015.

[32] Schué M, Maurin D, Dhouib R, N'Goma JC, Delorme V, Lambeau G, et al.
Two cutinase-like proteins secreted by Mycobacterium tuberculosis show very different lipolytic activities reflecting their physiological function. Faseb J. 2010;24:1893-903.

[33] Ravindran MS, Rao SP, Cheng X, Shukla A, Cazenave-Gassiot A, Yao SQ, et al. Targeting Lipid Esterases in Mycobacteria Grown Under Different Physiological Conditions Using Activitybased Profiling with Tetrahydrolipstatin (THL). Mol Cell Proteomics. 2014;13:43548.

[34] Liu Y, Patricelli MP, Cravatt BF. Activity-based protein profiling: The serine hydrolases. Proc Natl Acad Sci. 1999;96:14694-9.

[35] Aloulou A, Rodriguez JA, Fernandez $\mathrm{S}$, van Oosterhout $\mathrm{D}$, Puccinelli $\mathrm{D}$, Carrière F. Exploring the specific features of interfacial enzymology based on lipase studies. Biochimica et Biophysica Acta (BBA) - Molecular and Cell Biology of Lipids. 2006;1761:995-1013.

[36] Pappin DJC, Hojrup P, Bleasby AJ. Rapid identification of proteins by peptidemass fingerprinting. Curr Biol. 1993;3:32732.

[37] Viljoen A, Richard M, Nguyen PC, Fourquet P, Camoin L, Paudal RR, et al. Cyclipostins and Cyclophostin analogs inhibit the antigen $85 \mathrm{C}$ from Mycobacterium tuberculosis both in vitro and in vivo. J Biol Chem. 2018.

[38] Belisle JT, Vissa VD, Sievert T, Takayama K, Brennan PJ, Besra GS. Role of the major antigen of Mycobacterium tuberculosis in cell wall biogenesis. Science. 1997;276:1420-2.

[39] Jackson M, Raynaud C, Laneelle MA, Guilhot C, Laurent-Winter C, Ensergueix $\mathrm{D}$, et al. Inactivation of the antigen $85 \mathrm{C}$ gene profoundly affects the mycolate content and alters the permeability of the Mycobacterium tuberculosis cell envelope. Mol Microbiol. 1999;31:1573-87.

[40] Claxton HB, Akey DL, Silver MK, Admiraal SJ, Smith JL. Structure and functional analysis of RifR, the type II 
thioesterase from the rifamycin biosynthetic pathway. J Biol Chem. 2009;284:5021-9. [41] Krissinel E, Henrick K. Inference of Macromolecular Assemblies from Crystalline State. Journal of Molecular Biology. 2007;372:774-97.

[42] Carr PD, Ollis DL. Alpha/beta hydrolase fold: an update. Protein Pept Lett. 2009; 16:1137-48.

[43] Martinez C, Nicolas A, van Tilbeurgh H, Egloff M-P, Cudrey C, Verger R, et al. Cutinase, a lipolytic enzyme with a preformed oxyanion hole. Biochemistry. 1994;33:83-9.

[44] Khan FI, Lan D, Durrani R, Huan W, Zhao Z, Wang Y. The Lid Domain in Lipases: Structural and Functional Determinant of Enzymatic Properties. Front Bioeng Biotechnol. 2017;5:16.

[45] van Tilbeurgh H, Egloff M-P, Martinez C, Rugani N, Verger R, Cambillau C. Interfacial activation of the lipaseprocolipase complex by mixed micelles revealed by X-Ray crystallography. Nature. 1993;362:814-20.

[46] Holm L, Rosenstrom P. Dali server: conservation mapping in 3D. Nucleic Acids Res. 2010;38:W545-9.

[47] Roussel A, Canaan S, Egloff MP, Riviere M, Dupuis L, Verger R, et al. Crystal structure of human gastric lipase and model of lysosomal acid lipase, two lipolytic enzymes of medical interest. J Biol Chem. 1999;274:16995-7002.

[48] Roussel A, Miled N, Berti-Dupuis L, Riviere M, Spinelli S, Berna P, et al. Crystal structure of the open form of dog gastric lipase in complex with a phosphonate inhibitor. J Biol Chem. 2002;277:2266-74.

[49] Aggarwal A, Parai MK, Shetty N, Wallis D, Woolhiser L, Hastings C, et al. Development of a Novel Lead that Targets M. tuberculosis Polyketide Synthase 13. Cell. 2017;170:249-59 e25.

[50] Comas I, Coscolla M, Luo T, Borrell S, Holt KE, Kato-Maeda M, et al. Out-ofAfrica migration and Neolithic coexpansion of Mycobacterium tuberculosis with modern humans. Nature Genetics. 2013;45:1176.
[51] Ferraris D, Miggiano R, Rossi F, Rizzi M. Mycobacterium tuberculosis Molecular Determinants of Infection, Survival Strategies, and Vulnerable Targets. Pathogens. 2018;7:17.

[52] Wellington S, Hung DT. The expanding diversity of Mycobacterium tuberculosis drug targets. ACS Infect Dis. 2018:DOI: 10.1021/acsinfecdis.7b00255.

[53] Yuan T, Sampson NS. Hit Generation in TB Drug Discovery: From Genome to Granuloma. Chem Rev. 2018.

[54] Zumla A, Nahid P, Cole ST. Advances in the development of new tuberculosis drugs and treatment regimens. Nat Rev Drug Discov. 2013;12:388-404.

[55] Miled N, Roussel A, Bussetta C, BertiDupuis L, Riviere M, Buono G, et al. Inhibition of dog and human gastric lipases by enantiomeric phosphonate inhibitors: a structure-activity study. Biochemistry. 2003;42:11587-93.

[56] Sciara G, Blangy S, Siponen M, Mc Grath S, van Sinderen D, Tegoni M, et al. A topological model of the baseplate of lactococcal phage Tuc2009. J Biol Chem. 2008;283:2716-23.

[57] Delorme V, Diomande SV, Dedieu L, Cavalier JF, Carriere F, Kremer L, et al. MmPPOX Inhibits Mycobacterium tuberculosis Lipolytic Enzymes Belonging to the Hormone-Sensitive Lipase Family and Alters Mycobacterial Growth. PLoS One. 2012;7:e46493.

[58] Verger R, de Haas GH. Interfacial enzyme kinetics of lipolysis. Annual Review Biophys Bioeng. 1976;5:77-117.

[59] Ransac S, Riviere C, Soulie JM, Gancet C, Verger R, de Haas GH. Competitive inhibition of lipolytic enzymes. I. A kinetic model applicable to water-insoluble competitive inhibitors. Biochimica et Biophysica Acta (BBA) Molecular and Cell Biology of Lipids. 1990;1043:57-66.

[60] Shevchenko A, Wilm M, Vorm O, Mann M. Mass spectrometric sequencing of proteins silver-stained polyacrylamide gels. Anal Chem. 1996;68:850-8. 
[61] Mueller U, Nyarsik L, Horn M, Rauth $\mathrm{H}$, Przewieslik T, Saenger $\mathrm{W}$, et al. Development of a technology for automation and miniaturization of protein crystallization. J Biotechnol. 2001;85:7-14. [62] Kabsch W. Xds. Acta Crystallogr D Biol Crystallogr. 2010;66:125-32.

[63] Adams PD, Afonine PV, Bunkoczi G, Chen VB, Davis IW, Echols N, et al. PHENIX: a comprehensive Python-based system for macromolecular structure solution. Acta Crystallogr D Biol Crystallogr. 2010;66:213-21.

[64] Emsley P, Cowtan K. Coot: modelbuilding tools for molecular graphics. Acta
Crystallogr D Biol Crystallogr. 2004;60:2126-32.

[65] Blanc E, Roversi P, Vonrhein C, Flensburg C, Lea SM, Bricogne G. Refinement of severely incomplete structures with maximum likelihood in BUSTER-TNT. Acta Crystallogr D Biol Crystallogr. 2004;60:2210-21.

[66] Lebedev AA, Young P, Isupov MN, Moroz OV, Vagin AA, Murshudov GN. JLigand: a graphical tool for the CCP4 template-restraint library. Acta Crystallogr D Biol Crystallogr. 2012;68:431-40. 
1 Table 1. Data collection and refinement statistics (numbers in brackets refer to the highest

2 resolution bin)

\begin{tabular}{|c|c|c|}
\hline $\begin{array}{c}\text { DATA } \\
\text { COLLECTION* }\end{array}$ & Form1 & Form2 \\
\hline PDB & $6 \mathrm{FW} 5$ & $6 \mathrm{FVJ}$ \\
\hline Source & SOLEIL, PROXIMA 1 & SOLEIL, PROXIMA 1 \\
\hline Detector & PILATUS 6M & PILATUS 6M \\
\hline Wavelength $(\AA)$ & 0.978570 & 0.97934 \\
\hline Space group & $\mathrm{F} 222$ & $\mathrm{C} 222_{1}$ \\
\hline cell $(\AA)$ & $\mathrm{a}=77.58, \mathrm{~b}=224.65, \mathrm{c}=226.62$ & $\mathrm{a}=79.07, \mathrm{~b}=222.58, \mathrm{c}=222.21$ \\
\hline Angles $\left(^{\circ}\right)$ & $\alpha=\beta=\gamma=90$ & $\alpha=\beta=\gamma=90$ \\
\hline Nr. of monomers & 4 & 8 \\
\hline Resolution limits $(\AA)$ & $43.87-2.75(2.91-2.75)$ & $43.80-2.60(2.75-2.6)$ \\
\hline Rmerge & $0.106(1.001)$ & $0.116(0.907)$ \\
\hline $\mathrm{CC} 1 / 2$ & $0.997(0.743)$ & $0.999(0.773)$ \\
\hline Unique reflections & $24307(4055)$ & $61244(9662)$ \\
\hline $\operatorname{Mean}((\mathrm{I}) / \mathrm{sd}(\mathrm{I}))$ & $10.00(1.38)$ & $15.94(2.43)$ \\
\hline Completeness (\%) & $93.1(97.1)$ & $99.6(98.8)$ \\
\hline Multiplicity & $7.18(7.19)$ & $13.4(13.5)$ \\
\hline \multicolumn{3}{|l|}{ REFINEMENT* } \\
\hline Resolution $(\AA)$ & $39.89-2.75(2.87-2.75)$ & $43.8-2.6(2.67-2.6)$ \\
\hline Number of reflections & $24306(3026)$ & $61125(4464)$ \\
\hline $\begin{array}{c}\text { Number of protein / } \\
\text { water / ligand (atoms) }\end{array}$ & $6766 / 80 / 20$ & $12774 / 327 / 157$ \\
\hline Test set reflections & $1216(152)$ & $3056(223)$ \\
\hline $\mathrm{R}_{\mathrm{work}} / \mathrm{R}_{\text {free }}(\%)$ & $27.2 / 28.1(29.4 / 31.2)$ & $23.4 / 25.4(25.6 / 25.3)$ \\
\hline $\begin{array}{l}\text { r.m.s.d.bonds }(\AA) / \\
\text { angles }\left({ }^{\circ}\right)\end{array}$ & $0.011 / 1.23$ & $0.008 / 1.04$ \\
\hline B-Wilson / B-mean $\AA$ & $145.61 / 133.02$ & $85.66 / 85.27$ \\
\hline $\begin{array}{l}\text { Ramachandran: } \\
\text { preferred / allowed / } \\
\text { outliers (\%) }\end{array}$ & $92.17 / 6.56 / 1.27$ & $94.44 / 3.89 / 1.67$ \\
\hline $\begin{array}{c}\text { Average B-factor per } \\
\text { chain: } \\
\text { A/B/C/D/E/F/G/H }\end{array}$ & $119.2 / 128.2 / 142.7 / 145.7$ & 72.2/67.6/74.8/67.2/87.9/91.2/113.8/112.3 \\
\hline
\end{tabular}



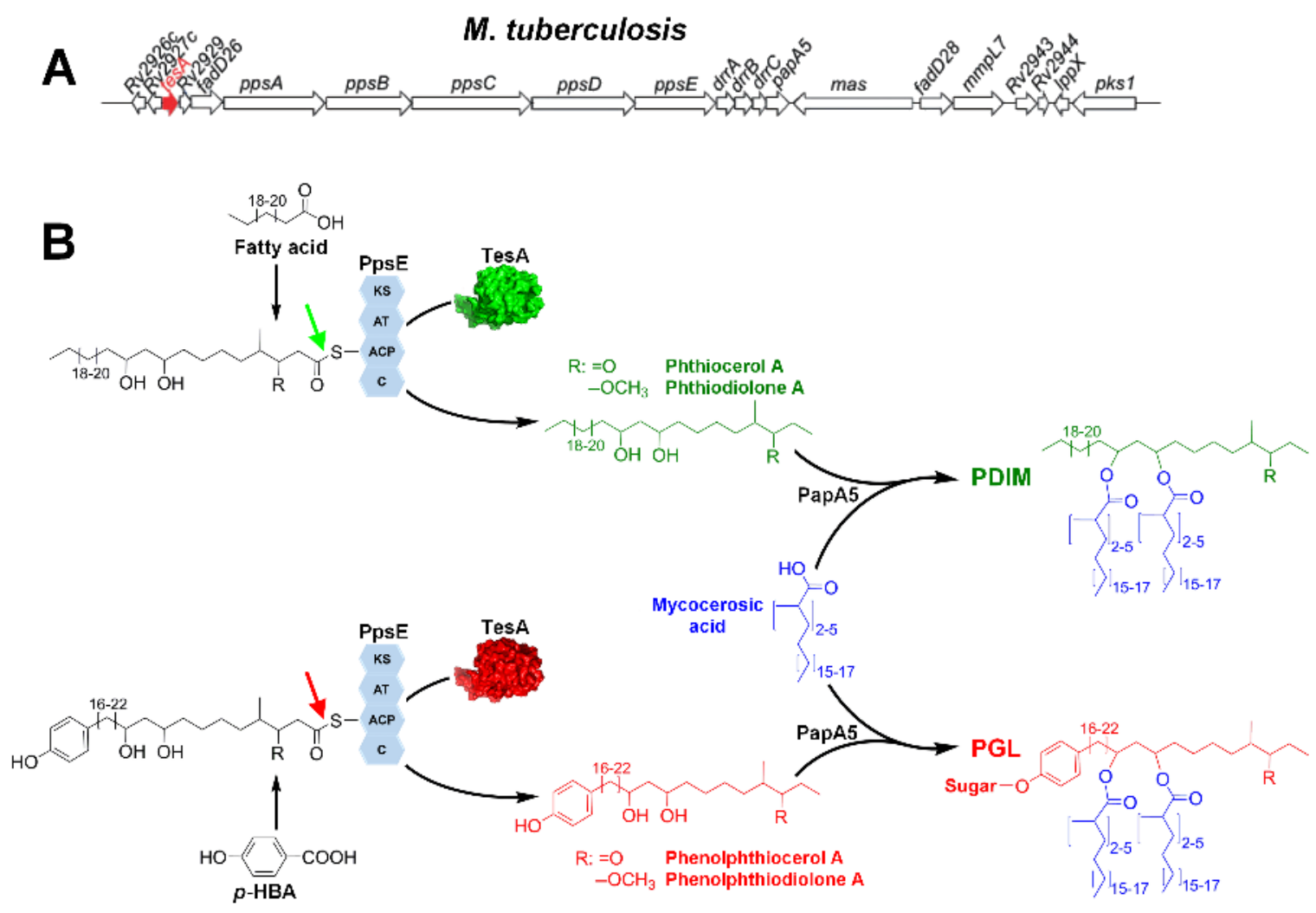

1

3

4

5

6

7

8

9

10

Figure 1: (A) The PDIM gene cluster in M. tuberculosis. The tesA genes is shown in red. The black arrows represent predicted open reading. (B) Schematic representation of phthiocerol dimycocerosate (PDIM) and phenolphthiocerol (PGL) biosynthesis in M. tuberculosis. The interaction between TesA and PpsE is represented. The hydrolyzed thioester bond is indicated by a green arrow in PDIM synthesis pathway, and by a red arrow in PGL biosynthesis. After condensation with mycoseric acid, the products released by TesA lead to the synthesis of both PDIM and PGL. 

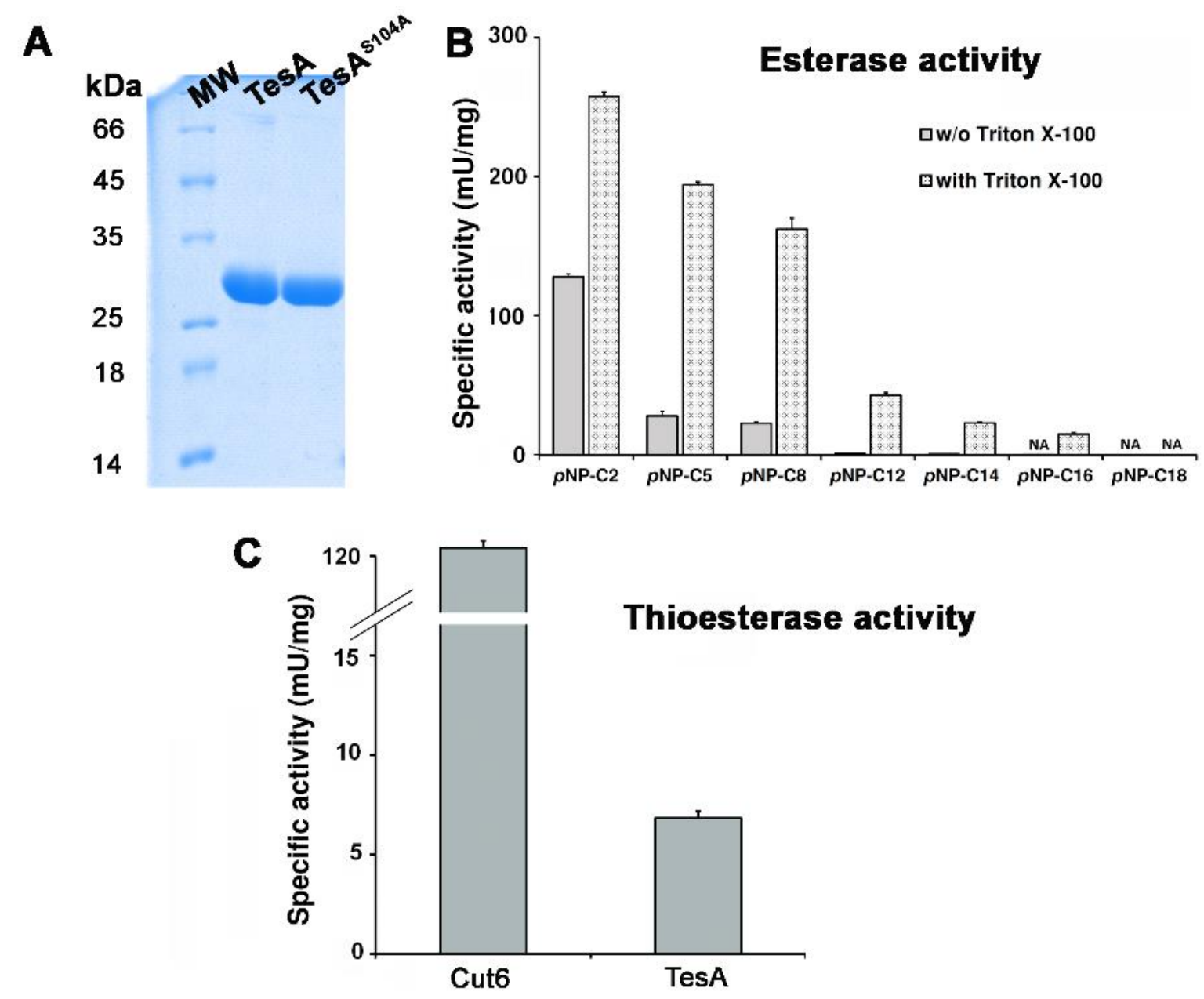

3

4

5

6

7

8

9

10

11

12

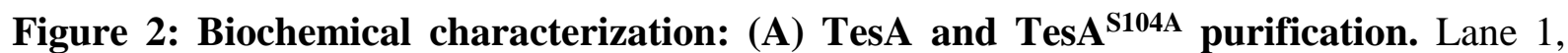
standard from Euromedex; lane 2 and 3, $3 \mu \mathrm{g}$ of purified TesA and TesA ${ }^{\text {S104A }}$ mutant after Tev proteolysis. (B) Esterase activity on $p$ Nitrophenyl esters with chain lengths varying from $\mathrm{C} 2$ to $\mathrm{C} 18$ in presence or in absence of $0.5 \%$ Triton X-100. (C) Thioesterase activity measurements corresponding to Palmitoyl-CoEnzyme A hydrolysis. Cut6 (Rv3802c) was used as positive control for the thioesterase activity. Each experiment is the mean of three independents assays. NA, no activity. 

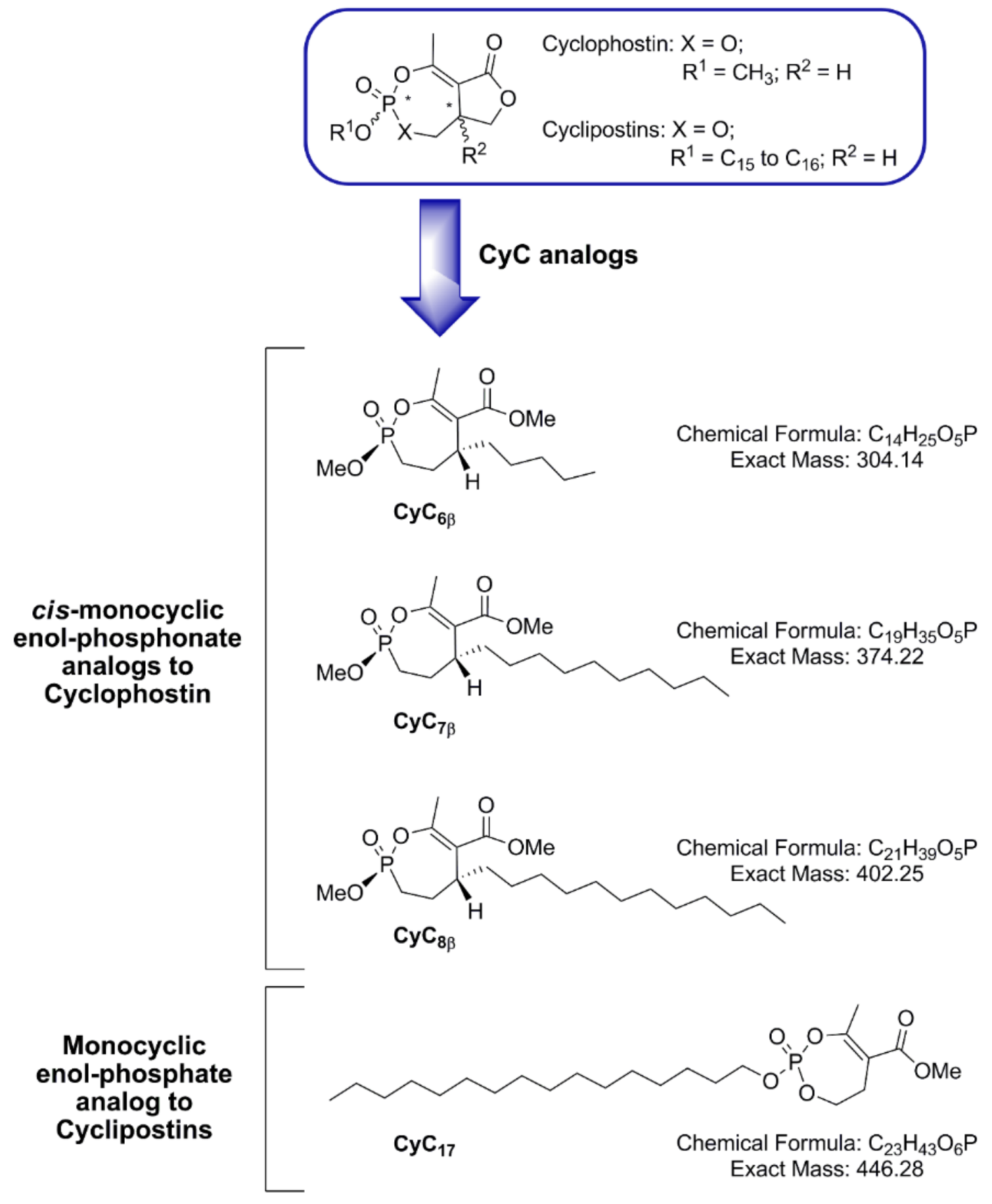

1

3

4

5

6

Figure 3: Chemical structures of $\mathrm{CyC}_{6 \beta}, \mathrm{CyC}_{7 \beta}, \mathrm{CyC}_{8 \beta}$ cis-monocyclic enol phosphonate analogs to Cyclophostin and $\mathrm{CyC}_{17}$ a monocyclic enol phosphate analog to Cyclipostins. Adapted from [19]. 


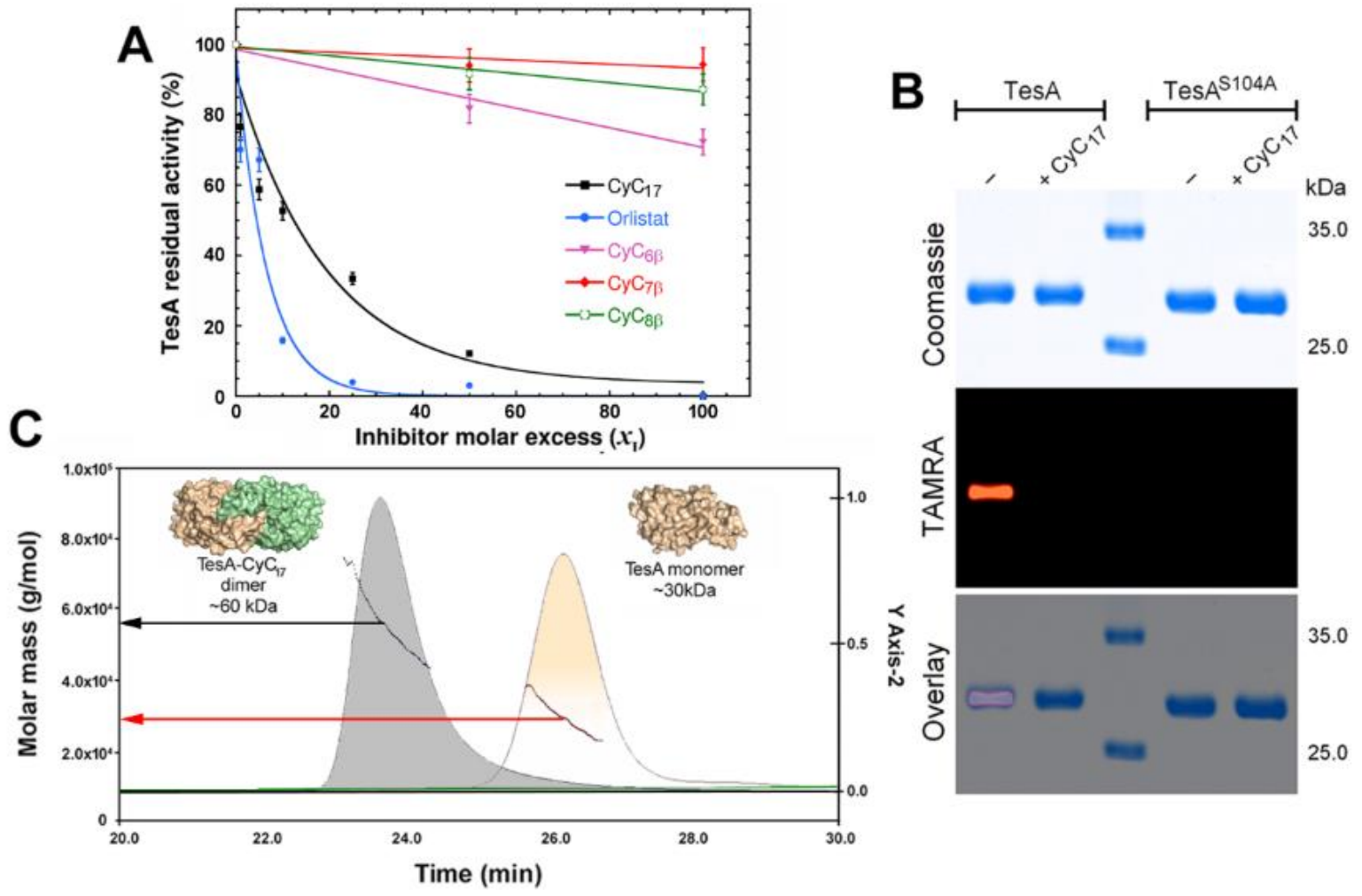

Figure 4: Inhibition of the TesA esterase activity is mediated by the covalent binding of CyC analogs. (A) The enzymatic activity of TesA was tested using $p \mathrm{NP}$ ester assay in the presence of different concentrations of $\mathbf{C y C}_{7 \beta}, \mathbf{C y C}_{8 \beta}, \mathbf{C y C}_{17}$ and Orlistat as control. The inhibitory effect was determined at the maximum rate of the reaction. Error bars represent the standard deviation calculated from three independent experiments. Dose-response curves for $\mathbf{C y C}_{7 \beta}, \mathbf{C y C}_{8 \beta}, \mathbf{C y C}_{17}$ and Orlistat were fitted in Kaleidagraph 4.2 Software (Synergy Software). (B) Equal amounts of either TesA or TesA ${ }^{\mathrm{S} 104 \mathrm{~A}}$ were pre-treated with $\mathbf{C y C}_{\mathbf{1 7}}$, incubated with TAMRA-FP, separated by SDS-PAGE and visualized by Coomassie blue staining (upper panel) or in-gel fluorescence visualization (middle panel). The merged image is shown in the lower panel. TAMRA labelling of TesA is blocked by the covalent binding of the $\mathbf{C y C}$ analogs to the catalytic Ser104 as evidenced with the TesA ${ }^{\mathrm{S} 104 \mathrm{~A}}$ variant. (C) MALS/QELS/UV/RI analysis. The corresponding curve depicting the variation in UV absorbance at $280 \mathrm{~nm}$ as a function of time (min. after sample injection in the High Performance Liquid Chromatography system) for TesA (orange brown chromatogram) and TesA-CyC17 complex (grey chromatogram) have been superimposed and reported on the same chromatogram. The traces indicating the molar mass (indicated on the left, in Da) are shown on each peak by an arrow. Molecular masses of 31,800 Da (CV\% <5\%) for the monomer and $56,000 \mathrm{Da}(\mathrm{CV} \%<4 \%)$ for the dimer have been calculated by the ASTRA software (Wyatt Technology). 
A

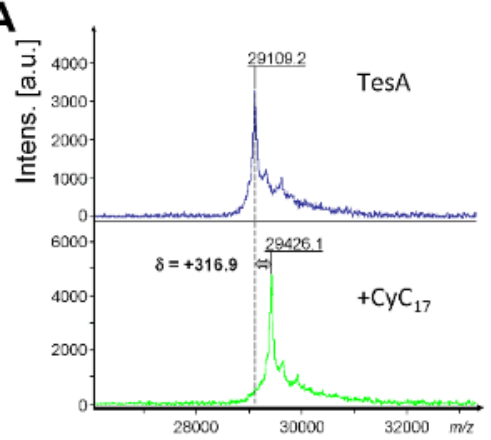

B

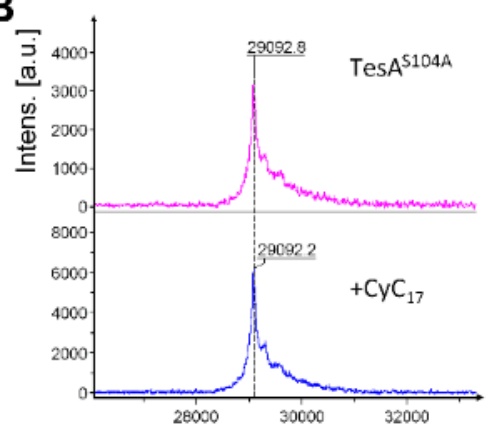

C

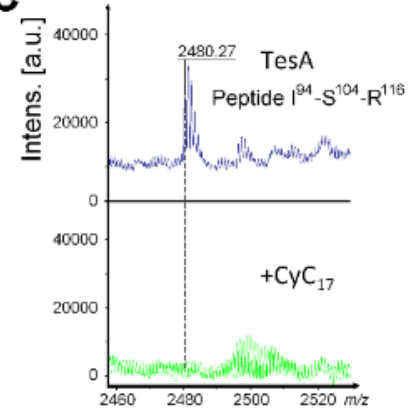

D

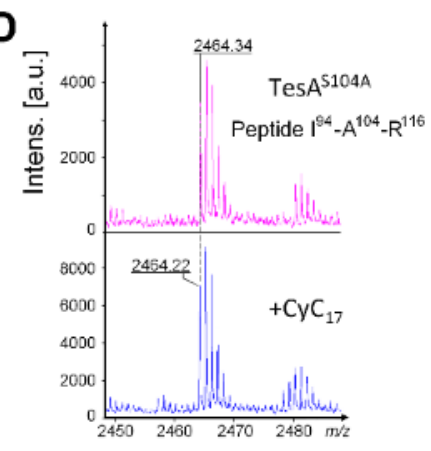

E
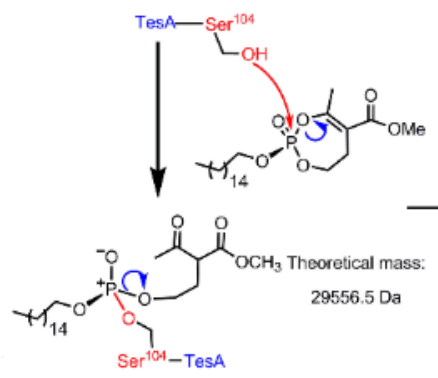

$\mathrm{H}_{2} \mathrm{O} \downarrow \mid \begin{gathered}\text { Phosphate ester } \\ \text { hydrolysis }\end{gathered}$

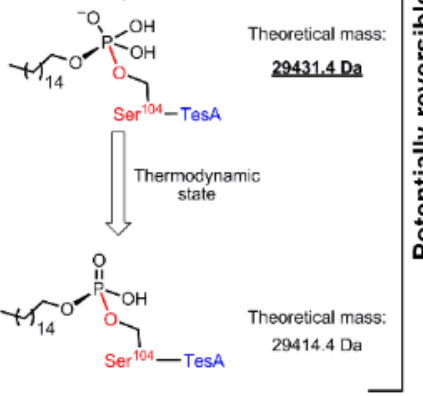

3

4

5

6

7

8

9

10

11

Figure 5: Mass spectrometry analyses. Global mass modification of TesA (A) and Tes $\mathrm{A}^{\mathrm{S} 104 \mathrm{~A}}$ (C) without (blue and pink spectra) or pre-incubated with $\mathbf{C y C}_{17}$ (green and blue spectra), as determined using an Ultraflex III mass spectrometer (Brucker Daltonics) in linear mode with the LP_66kDa_method. In order to localize the peptide containing the catalytic Ser104, TesA and TesA ${ }^{\text {S104A }}$ were digested by trypsin. (B) and (D), peptide mass fingerprint on the digested TesA/Tes $\mathrm{A}^{\mathrm{S} 104 \mathrm{~A}}$ (upper spectra, blue and pink), and peptide mass fingerprint on the digested TesA/Tes $\mathrm{A}^{\mathrm{S} 104 \mathrm{~A}}$ pre-treated by $\mathbf{C y C}_{\mathbf{1 7}}$ (lower spectra, green and blue). (E) Mechanism of action of the phosphate analog $\mathbf{C y C}_{\mathbf{1 7}}$ based on mass spectrometry analyses. 
Figure 6

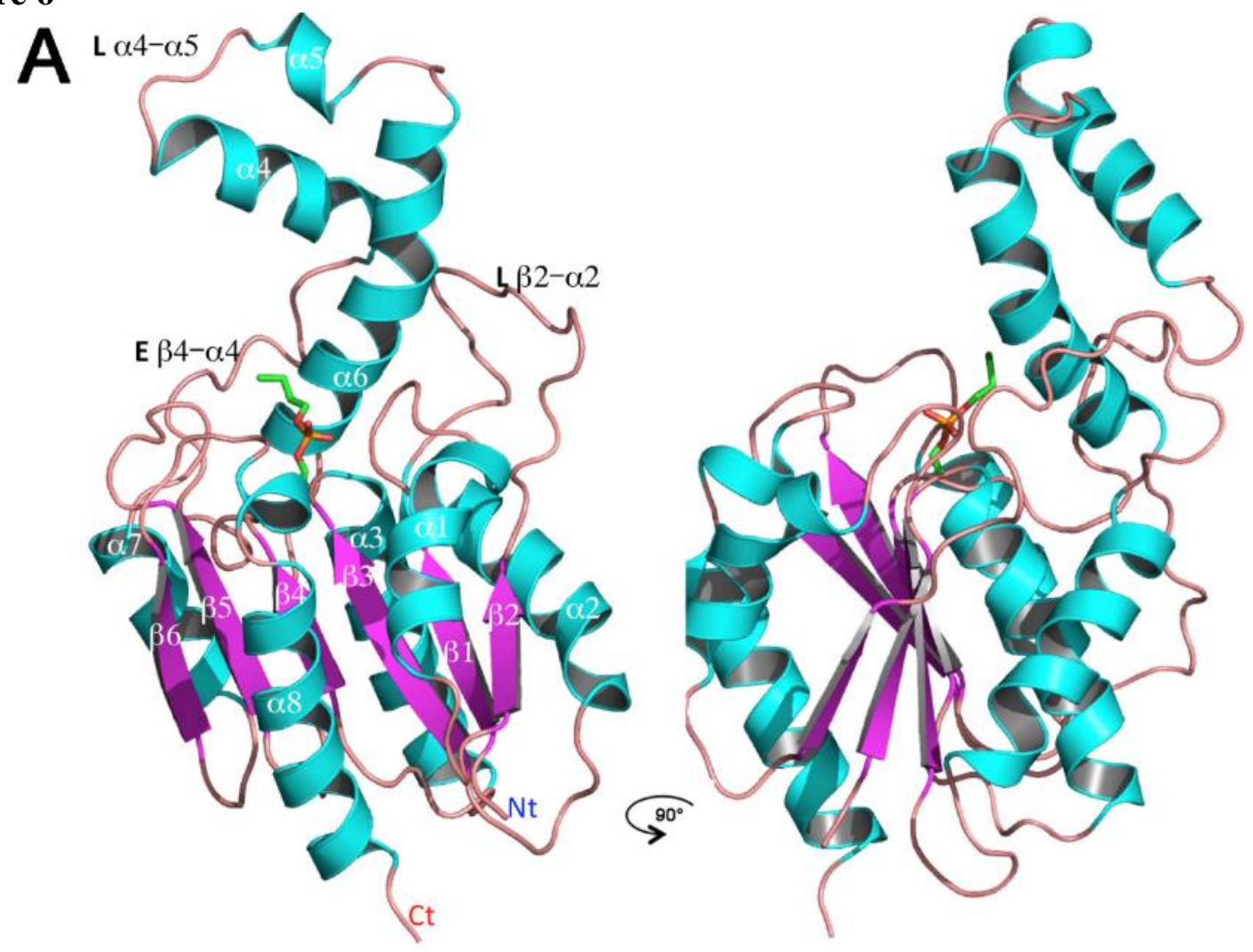

Figure 6: (A) Ribbon view of overall structure of TesA, face view and $90^{\circ}$ rotation around vertical axis. The $\alpha$-helices are colored cyan blue and the $\beta$-strands magenta. (B) The catalytic machinery of TesA. The catalytic triad Ser104-His236-Asp208 side chains are colored by atom type (N: blue, O: red, C: grey) with black labels. The hydrogen bonds are colored green. The oxyanion hole residues (Ala37 and Met105) are labelled in white with yellow colored hydrogen bonds. 

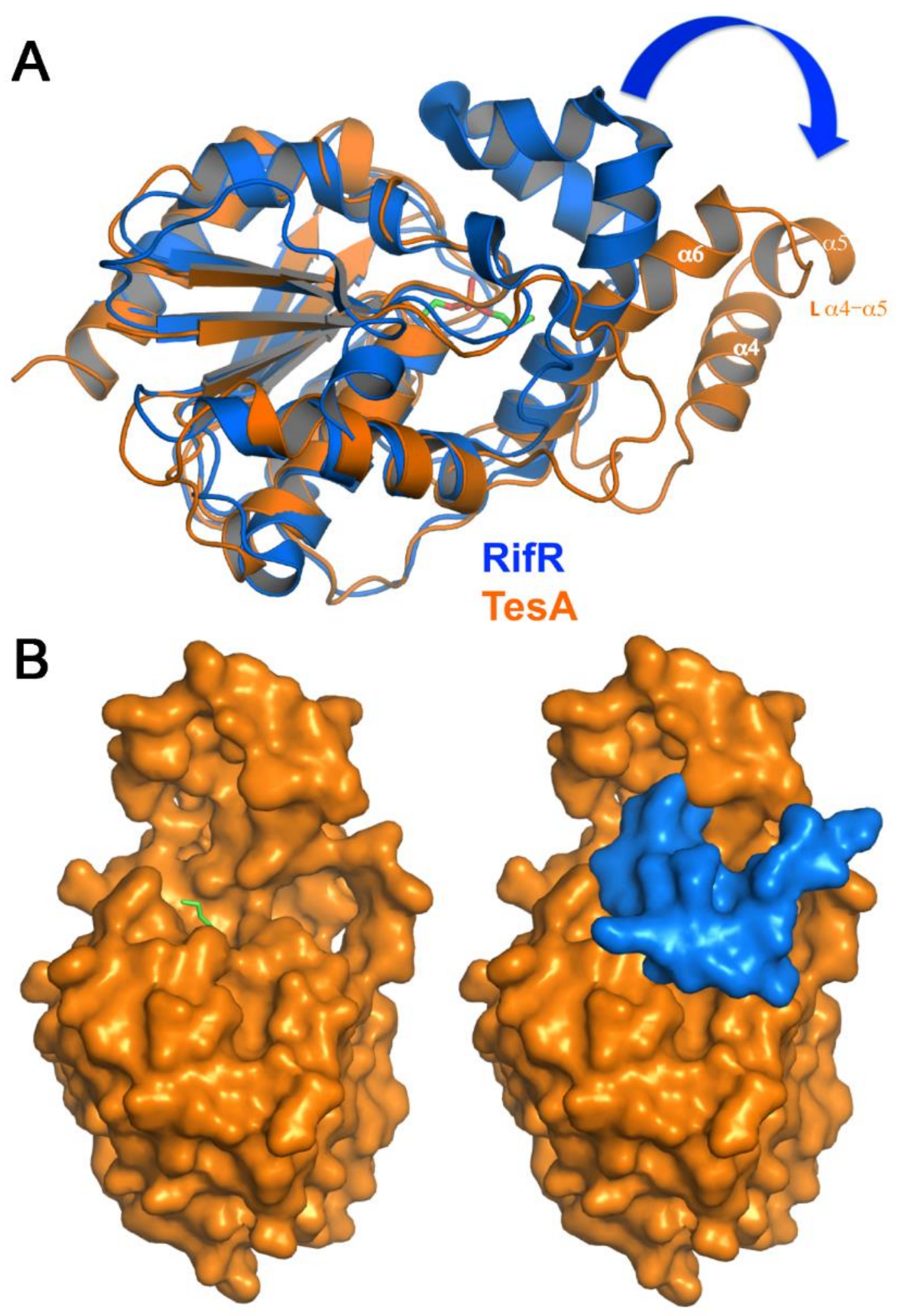

Figure 7: Identification of TesA lid. (A) Ribbon view of the superposition of TesA with RifR (PDB id: 3FLA; Type II thioesterase from Rifamycin NRPS/PKS biosynthetic pathway). TesA and RifR are colored in orange and blue, respectively. The hypothetical rotation from closed to open form is identified by a blue arrow. (B) Left: surface representation of TesA (orange) with the inhibitor $\mathbf{C y C}_{17}$ (stick representation) in the active site. Right: same as on the left, but with the ribbon view of RifR (PDB id: 3FLA) superimposed. The helices of RifR lid (blue color) cover the catalytic site of TesA. 

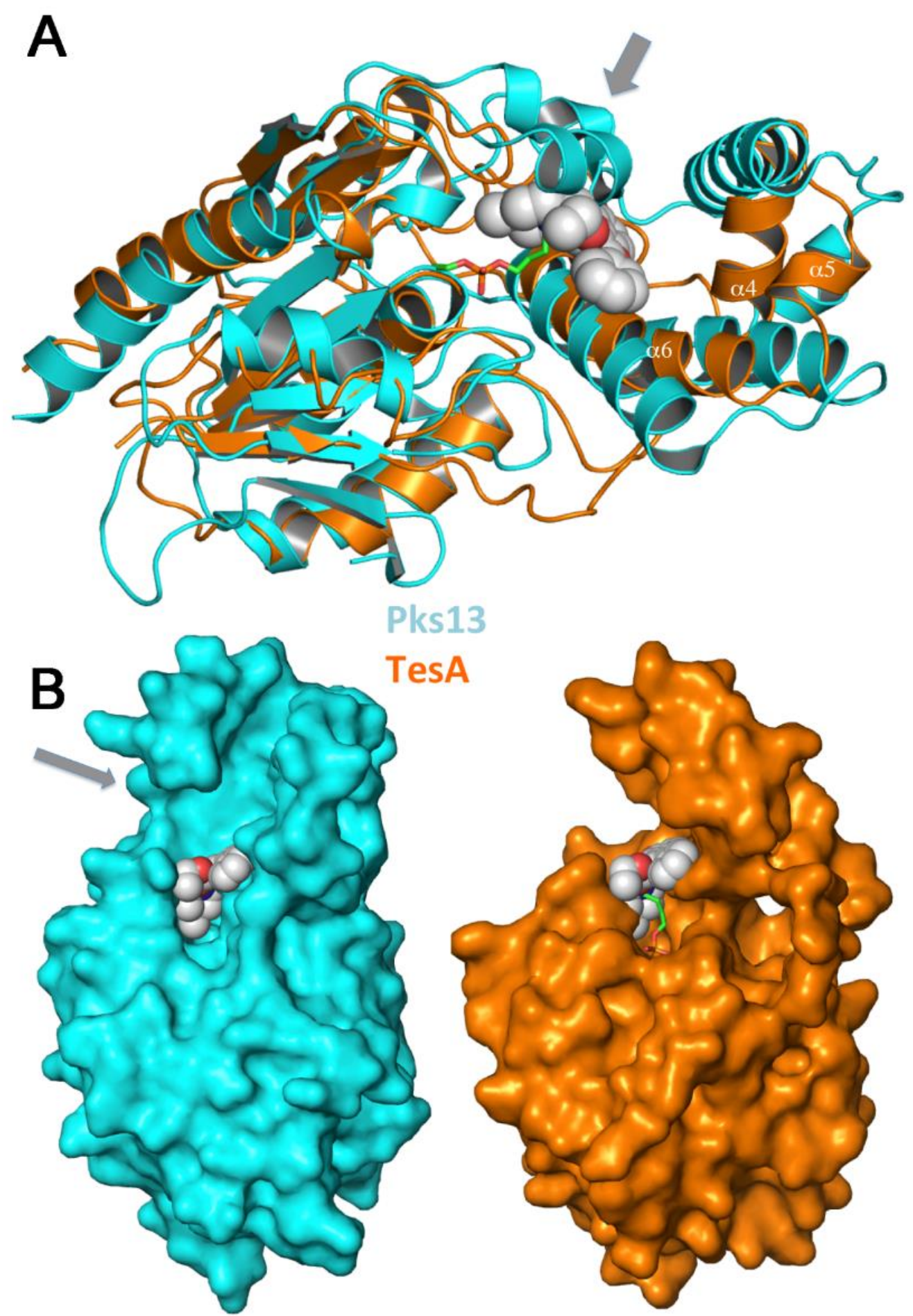

Figure 8: Comparison of TesA with the thioesterase domain of M. tuberculosis Polyketide Synthase 13 (Pks13). (A) Ribbon view of the superposition of TesA with the thioesterase domain of Pks13 (PDB id: 5V3X) colored orange and cyan blue, respectively. TesA and Pks13 inhibitors are represented as sticks and spheres, respectively. Both lids superimposed well and are in open conformation. The grey arrow identifies the two helices insertion in Pks13 thioesterase domain. (B) Left: Surface representation of Pks 13 thioesterase domain (cyan blue) and its inhibitor. Right: Surface representation of TesA (orange) and inhibitor $\mathbf{C y C} \mathbf{1 7}$. The grey arrow identifies the position of the two helices insertion in Pks13 thioesterase domain. 

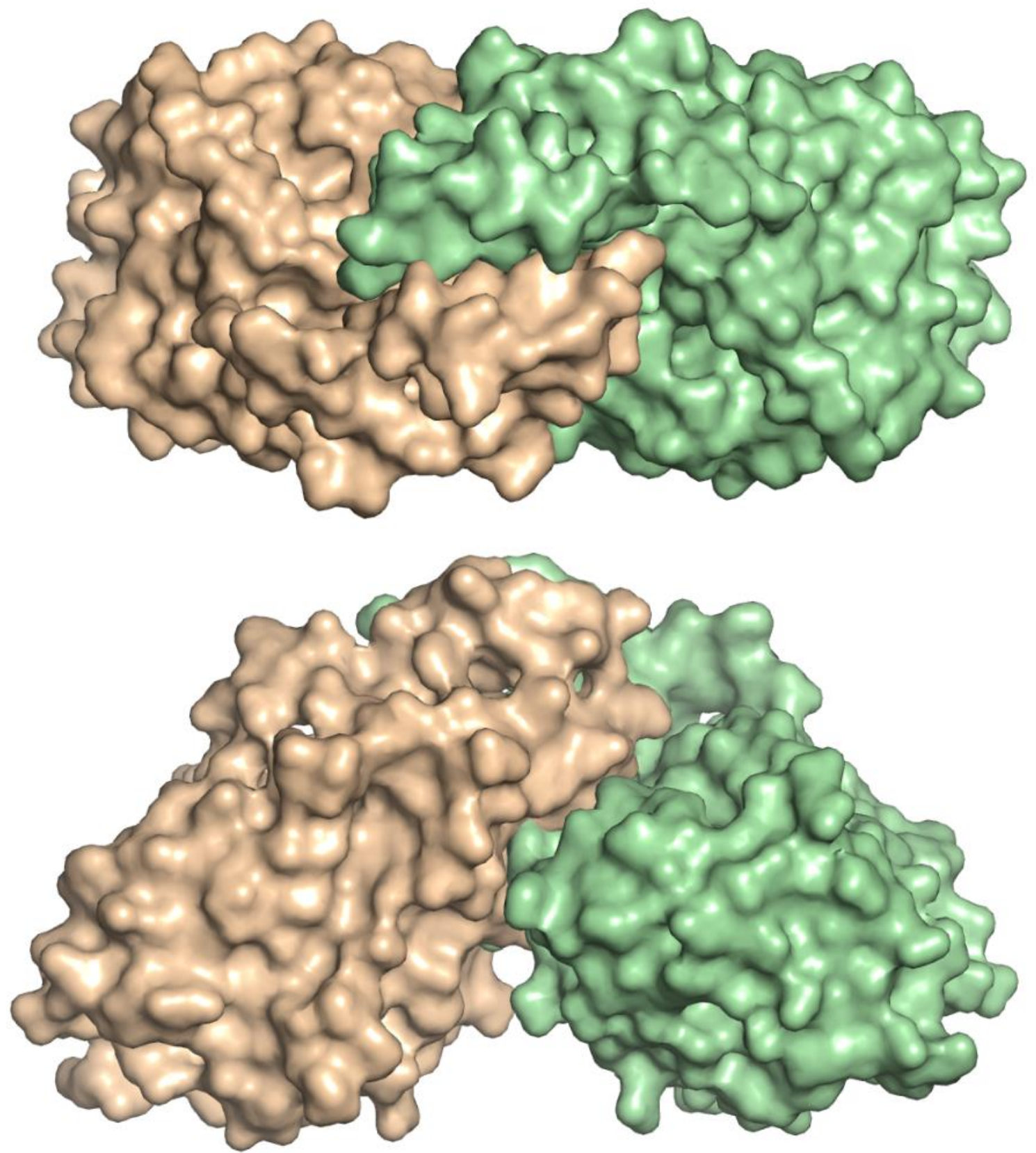

Figure S1: Dimer formation of TesA in presence of inhibitor $\mathrm{CyC}_{17}$ 
Figure S2

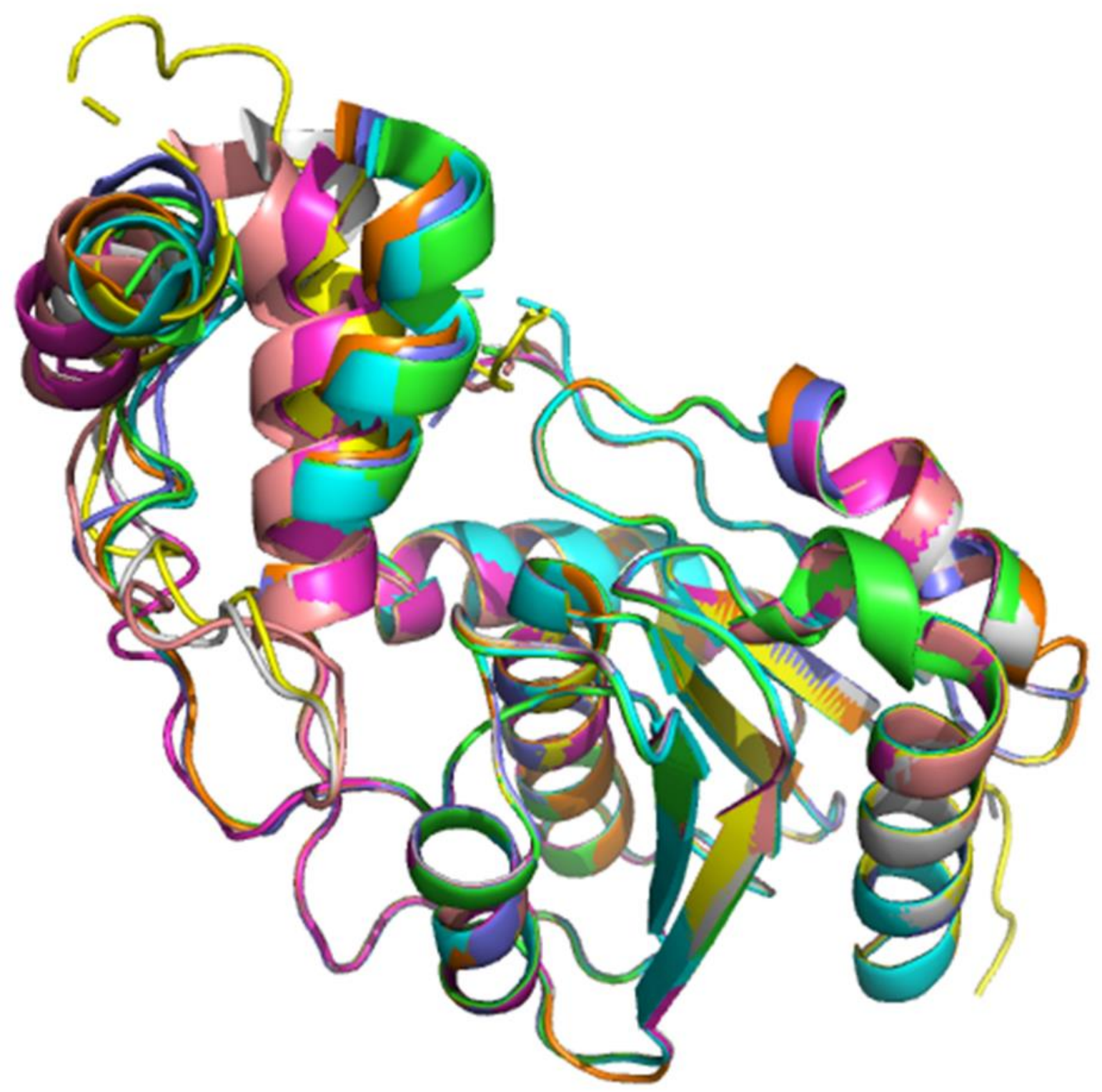

Figure S2: Superposition of 8 monomers of TesA identified in the asymmetric unit 
$1 \quad$ Figure S3

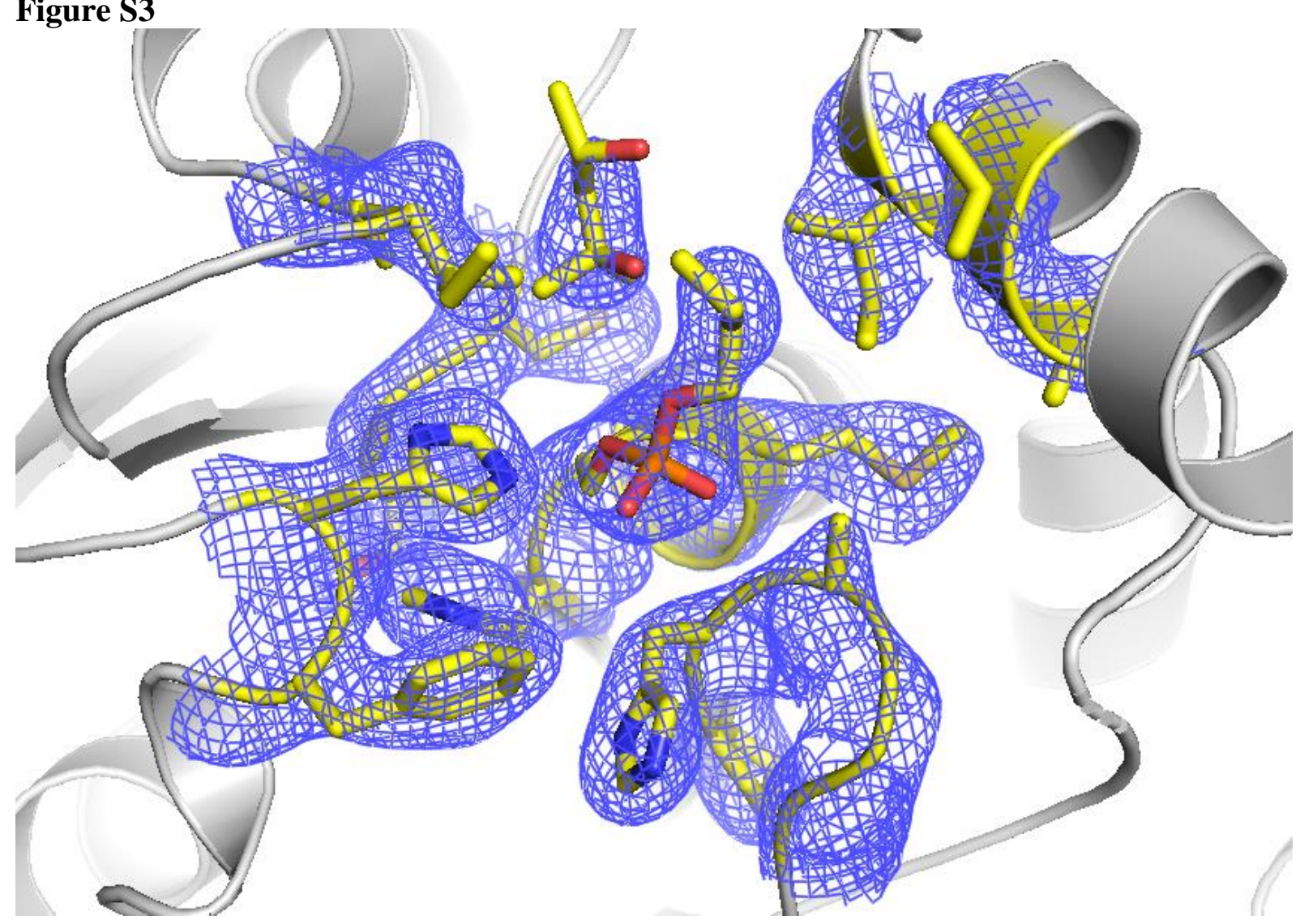

Figure S3: 2Fo-Fc electron density map contoured at $1 \sigma$ of the ligand $\left(\mathrm{CyC}_{17}\right)$ and residues within a $8 \AA$ radius from ligand to each atom 